\title{
Seed Nutrition and Quality, Seed Coat Boron and Lignin Are Influenced by Delayed Harvest in Exotically-Derived Soybean Breeding Lines under High Heat
}

\author{
Nacer Bellaloui ${ }^{1 *}$, James R. Smith ${ }^{1}$ and Alemu Mengistu ${ }^{2}$ \\ ${ }^{1}$ Crop Genetics Research Unit, USDA, Agricultural Research Service, Stoneville, MS, United States, ${ }^{2}$ Crop Genetics \\ Research Unit, USDA, Agricultural Research Service, Jackson, TN, United States
}

OPEN ACCESS

Edited by:

Gea Guerriero,

Luxembourg Institute of Science and Technology, Luxembourg

Reviewed by:

Hao Peng,

Washington State University,

United States

Sergio Esposito,

University of Naples Federico II, Italy

${ }^{*}$ Correspondence:

Nacer Bellalou

nacer.bellaloui@ars.usda.gov

Specialty section:

This article was submitted to

Plant Nutrition,

a section of the journa

Frontiers in Plant Science

Received: 14 July 2017

Accepted: 28 August 2017

Published: 22 September 2017

Citation:

Bellaloui N, Smith JR and Mengistu A (2017) Seed Nutrition and Quality, Seed Coat Boron and Lignin Are Influenced by Delayed Harvest in Exotically-Derived Soybean Breeding Lines under High Heat.

Front. Plant Sci. 8:1563.

doi: 10.3389/fpls.2017.01563
The timing of harvest is a major factor affecting seed quality in soybean, particularly in Midsouthern USA, when rain during harvest period is not uncommon. The objective of this research was to evaluate the effects of time of harvest on soybean seed quality (seed composition, germination, seed coat boron, and lignin) in high germinability (HG) breeding lines (50\% exotic) developed under high heat. The hypothesis was that seeds of HG lines possess physiological and genetic traits for a better seed quality at harvest maturity and delayed harvest. A 2-year field experiment was conducted under irrigated conditions. Results showed that, at harvest maturity, the exotic HG lines had higher seed protein, oleic acid, sugars, seed coat boron, and seed coat lignin, but lower seed oil compared with the non-exotic checks (Control), confirming our hypothesis. At 28 days after harvest maturity (delayed harvest), the content of seed protein, oleic acid, sugars, seed coat boron, and seed coat lignin were higher in some of the HG lines compared with the checks, indicating a possible involvement of these seed constituents, especially seed coat boron and seed coat lignin, in maintaining seed coat integrity and protecting seed coat against physical damage. Highly significant positive correlations were found between germination and seed protein, oleic acid, sugars, and seed coat boron and seed coat lignin. Highly significant negative correlation was found between germination and oil, linoleic acid, seed coat wrinkling, shattering, and hard seed. Yields of some HG lines were competitive with checks. This research demonstrated that time of harvesting is an important factor influencing seed protein and oil production. Also, since high oleic acid is desirable for oxidative stability, shelf-life and biodiesel properties, using HG lines could positively influence these important traits. This result should suggest to breeders of some of the advantages of selecting for high seed coat boron and lignin, and inform growers of the importance of timely harvest for maintaining high seed quality.

Keywords: soybean germinability, seed quality, seed nutrition, seed coat, boron, lignin, seed protein, seed oil 


\section{INTRODUCTION}

Soybean is an important crop in the world and is mainly produced in the USA (36.7\%), Brazil (31\%), Argentina (16\%), and China (4.8\%) (Belewu and Belewu, 2007; FAOSTAT, 2011). Seed of soybean is a source of soymeal for livestock, and oil for human consumption (Wilson, 2004), and recently, for biodiesel production (Baldoni et al., 2013; Santos et al., 2013). Soybean seed contain about $37-45 \%$ protein, 19 to $25 \%$ triglyceride oil composed of various fatty acids (palmitic: 9-14\%; stearic: 2-5\%; oleic: 19-28\%; linoleic: 48-58\%; and linolenic 5-11\%) (Wilson, 2004; Bellaloui et al., 2017). Soybean seed also contains sugars, such as sucrose, raffinose, stachyose, glucose, and fructose; mineral nutrients including $\mathrm{K}, \mathrm{Ca}, \mathrm{Fe}, \mathrm{Mg}, \mathrm{Mn}, \mathrm{Cu}, \mathrm{Zn}$, and $\mathrm{B}$; and phenolics such lignin and isoflavones (Sakthivelu et al., 2008; Bellaloui et al., 2009, 2010a,b, 2011). High seed protein and oil are desirable for human and livestock nutrition; high oleic and palmitic acids and low linolenic fatty acid are desirable for biodiesel and shelf-life due to oxidative stability of the oil (Ferrari et al., 2005; Atabania et al., 2012; Santos et al., 2013).

In the Midsouthern USA, especially in the Mississippi River Delta region, the Early Soybean Production System (ESPS) led to higher yield under irrigation and non-irrigation; however, the use of this system results in poor seed quality due to high heat, diseases, and early rain during harvest, leading to weathering effects (TeKrony et al., 1996; Mengistu and Heatherly, 2006; Smith et al., 2008; Bellaloui et al., 2012b,c,a). High temperature and delayed harvest, due to rain during harvest, is normal in ESPS, and these conditions lead to seed coat wrinkling and cracking, declining of seed coat physiological integrity, increasing seed deterioration and reducing seed quality (Franca-Neto et al., 1988, 1993).

Two major seed quality components have been reported to be associated with seed germination and seed quality: boron and seed coat lignin. Boron is a micronutrient essential for growth, development, yield and seed quality (Pilbeam and Kirkby, 1983; Marschner, 1995; Brown et al., 1999; Dordas, 2006; Dordas et al., 2007). Boron is also involved in cell wall structure and cell membrane integrity ( $\mathrm{Hu}$ and Brown, 1994; Brown et al., 2002), nitrogen metabolism (Shelp, 1993; Marschner, 1995), carbohydrate metabolism (Marschner, 1995), including sugar alcohols (Bellaloui et al., 1999; Brown et al., 1999), phenolic metabolism (Marschner, 1995), and nutrient uptake (Goldbach, 1985; Marschner, 1995). Lignin is a major factor influencing seed quality, including germination, shattering, hardseed, water permeability, and resistance to seed deterioration. For example, de Oliveira et al. (2014) concluded that higher lignin content in the pod and in the seeds may contribute to the decrease in seed deterioration from moisture. Alvarez et al. (1997) observed similar results among the cultivars, with the greatest lignin content in the seed coat for the seeds of the soybean cultivars. However, they also found a significant positive correlation between lignin content in the seed and stink bug damage, indicating that the lignin content in the pod or seed coat did not affect the pod walls resistance to insect.
Previous research showed that seed coat lignin (Krzyzanowski et al., 2008; de Oliveira et al., 2014) and seed B, seed protein, and seed oleic fatty acid, were associated with high seed quality (seed germination and vigor) (Bellaloui et al., 2017), but in those studies delaying harvest was not studied. Time of harvesting is a crop management factor that can significantly affect seed quality (Wilcox et al., 1974; Woodstock et al., 1985) and composition (Woodstock et al., 1985). Adverse weather conditions, such as frequent rain events prevent farmers from harvesting soybeans in the Midsouth USA or tropical regions (de Oliveira et al., 2014) on time, leading to seed quality deterioration (Jaureguy et al., 2013). So far, there are no cultivars with high germinability (HG) or heat tolerance available in the market, although the recent studies of Chebrolu et al. (2016) and Bellaloui et al. (2017) observed new exotically-derived breeding lines with improved seed quality under high heat environments. Therefore, developing breeding lines with high seed quality under high heat conditions of the Midsouthern USA, and understanding the physiology and genetic traits of seed quality responses to delayed harvest is critical and it is the focus of this research. The objective of this research was to evaluate exotically-derived soybean breeding lines with $\mathrm{HG}$ for their nutritional qualities and investigate the effects of delayed harvest on soybean seed nutrition quality components (seed chemical composition: protein, oil, fatty acids, sugars; seed coat B and lignin; and seed germination, hard seed, and shattering). To our knowledge, this is the first research to use exotic soybean breeding lines selected for high seed germinability and seed chemical nutrition in such a study. Since boron is known to be involved in seed germination and quality and has a structural role in cell walls and cell membrane (Marschner, 1995; Bellaloui and Brown, 1998), and involved in seed coat integrity and phenolics metabolism (Marschner, 1995), seed coat B and seed coat lignin was also given special attention.

\section{MATERIALS AND METHODS \\ Description of Experimental Breeding Lines}

The four exotically-derived breeding lines, two exotic accessions, and six non-exotic checks (four cultivars and two released germplasm lines) evaluated in this study are shown in Table 1. Breeding lines 25-1-1-1-1-4, 30-1-4-1-1, and 34-3-1-2-4-1 were derived from PI 587982A, which was identified by Smith et al. (2008) to have HG. The other parents for each of the above three lines were DT98-9102 (released by USDA-ARS in December 2004, but not entered into GRIN), "5601T" (Pantalone et al., 2003), and DT97-4290 (Paris et al., 2006), respectively. Breeding line 24-2-1-2-1-2 was derived from DT98-9102 × PI603756. The PI 603756, with HG, was also identified by Smith et al. (2008). Each line of the above breeding lines has 50\% exotic parentage. One public cultivar from Illinois LD00-3309 (Diers et al., 2006) was included in the study; and two commercial cultivars (AG4903 developed by the Monsanto Corporation and 94B73 developed by DuPont/Pioneer) were also included. It should be noted that all parents of the four breeding lines were included in this study (5601T, DT97-4290, PI587982A, and PI603756). The genotypes used in the study were categorized into two groups: 1- breeding 
TABLE 1 | Maturity, germination, and yield of genotypes group by their levels of exotic parentage grown under high heat of Mississippi Delta conditions in a 2-year field experiment to test for the effects of delayed harvesting/time of harvest on seed nutrition and quality.

\begin{tabular}{|c|c|c|c|c|c|c|c|}
\hline \multirow[t]{3}{*}{ Genotype } & \multirow[t]{3}{*}{ Maturity group } & \multicolumn{2}{|c|}{ Days to full maturity (R8) } & \multicolumn{2}{|c|}{ Germination (\%) } & \multicolumn{2}{|c|}{ Yield (kg ha-1) } \\
\hline & & 2010 & 2011 & 2010 & 2011 & 2010 & 2011 \\
\hline & & Days & Days & & & & \\
\hline \multicolumn{8}{|l|}{ HG (EXOTIC) } \\
\hline $34-3-1-2-4-1$ & III & 133 & 146 & 92.7 & 92.0 & 3,306 & 2,825 \\
\hline $30-1-4-1-1$ & III & 119 & 142 & 96.0 & 93.0 & 2,724 & 2,219 \\
\hline $25-1-1-1-1-4$ & IV & 152 & 164 & 92.7 & 91.0 & 3,934 & 2,914 \\
\hline $24-2-1-2-1-2$ & IV & 154 & 164 & 83.7 & 90.0 & 4,006 & 3,383 \\
\hline Pl603756 & $\|$ & 103 & 119 & 86.0 & 95.7 & 1,222 & 444 \\
\hline PI587982A & III & 122 & 140 & 95.7 & 81.3 & 1,840 & 1,446 \\
\hline \multicolumn{8}{|l|}{ CHECKS } \\
\hline LD00-3309 & IV & 133 & 146 & 37.3 & 78.0 & 3,347 & 3,268 \\
\hline DT98-9102 & IV & 165 & 166 & 88.7 & 78.3 & 3,183 & 3,051 \\
\hline DT97-4290 & IV & 161 & 166 & 40.7 & 87.7 & 3,930 & 3,697 \\
\hline AG4903 & IV & 161 & 166 & 44.0 & 87.3 & 4,528 & 3,376 \\
\hline 94B73 & IV & 142 & 154 & 56.7 & 76.3 & 3,901 & 4,566 \\
\hline $5601 \mathrm{~T}$ & V & 172 & 174 & 75.7 & 46.0 & 3,136 & 749 \\
\hline LSD & & & & 3.7 & 10.8 & 221 & 97 \\
\hline
\end{tabular}

lines derived from exotic parental accessions and previously identified to have HG under irrigation in the ESPS (25-1-1-1-14, 34-3-1-2-4-1, 30-1-4-1-1, and 24-2-1-2-1-2) and their exotic parents (PI587982A and PI603756); and 2- cultivars (LD003309, 5601T, AG4903, and 94B73) and released germplasm lines (DT97-4290 and DT98-9102).

\section{Field Management and Growth Conditions}

A 2-year field experiment was conducted in 2010 and 2011, and planting dates were on 6 April. The experiments were conducted at the Jamie Whitten Delta States Research Center in Stoneville, MS, USA. Each experiment unit was a 4-row plots with a 0.91 $\mathrm{m}$ row spacing, and plots were $5.79 \mathrm{~m}$ long at planting. The plots were trimmed to $4.88 \mathrm{~m}$ long after R1 (beginning bloom and before R6 (full seed) (Fehr and Caviness, 1977). The middle two rows of each plot were harvested with a combine (Almaco, IL, USA) shortly after R8 (full maturity) and weighed for seed yield. We used the term "harvest maturity" to refer to the time of harvest shortly after R8. Fourteen days after the initial harvest of each plot, one border row was hand-harvested and threshed with a bundle thresher. Twenty-eight days after the initial harvest of each plot, the second border row was hand-harvested and threshed as before. Harvested seed was stored at $21^{\circ} \mathrm{C}$ and $60 \%$ relative humidity until assayed for seed quality components. Seed characteristics (composition, seed vigor, hardseededness, and germinability) were made on each replicate. Although the maturity groups (MG) ranged from II to V, the field was designed as such all plots were harvested by the combine as they matured. All plots were furrow irrigated every 7-10 days throughout the growing season as needed to avoid any potential drought stress. After R8 and before initial harvest, plant height, shattering, and lodging were recorded. Physical and chemical analyses indicated that the soil was clay and had adequate soil nutrients. Boron concentration in soil was $2.5 \mathrm{mg} \mathrm{kg}^{-1}$, indicating that there was no boron deficiency in the soil. Leaves did not show any nutrient deficiency symptoms.

\section{Seed Analysis for Protein, Oil, Fatty Acids, and Sucrose}

Samples from mature seeds at initial harvest (harvested shortly after full maturity; $\mathrm{H} 1$; we have referred to as harvest maturity) and at delayed harvests ( 14 and 28 days after the initial harvest; $\mathrm{H} 2$ and $\mathrm{H} 3$, respectively) were analyzed for seed protein, oil, fatty acids, and sugars. About $25 \mathrm{~g}$ from each seed sample were ground by a Laboratory Mill 3600 (Perten, Springfield, IL), and protein, oil, fatty acids were analyzed by near infrared reflectance (Wilcox and Shibles, 2001) using a diode array feed analyzer AD 7200 (Perten, Springfield, IL) as detailed by Bellaloui et al. (2009) and Bellaloui et al. (2017). The analysis of the samples was performed based on the reference samples according to AOAC methods for protein and oil. The content of protein in seeds was estimated by measuring total nitrogen using the Kjeldahl method. Protein was calculated from total nitrogen using Dumas, Nx6.25 (AOAC, 1990a). For oil content in seeds, the oil was determined using the Soxhlet extraction method (AOAC, 1990b). Fatty acid analysis was based on reference samples determined by gas chromatography using fatty acids methyl esters (FAME) method (AOAC, 2000). The preparation and analysis of FAME were conducted by direct esterification with methanolic soldium methoxide. The separation was conducted by capillary gas chromatography, and the identification was determined by comparing the samples to pure standards. Calibrations were developed by the University of Minnesota, using Perten's Thermo Galactic Grams PLS IQ software, and established according to AOAC methods (AOAC, 1990a,b) and AOAC (2000). Measurement of protein and oil were reported as a percentage of seed dry matter basis (Wilcox and 
Shibles, 2001; Boydak et al., 2002) and fatty acids were based on total oil.

For sucrose a similar procedure of seed grinding was followed as indicated above. Briefly, about $25 \mathrm{~g}$ of seed from each replicate were ground by a Laboratory Mill 3600 (Perten, Springfield, IL). Seed sucrose content was determined by NIR (Wilcox and Shibles, 2001; Bellaloui et al., 2010b). The calibration equation was developed by the Department of Agronomy and Plant Genetics, University of Minnesota St Paul, MN, using Thermo Galactic Grams PLS IQ software, and developed by Perten company (Perten, Springfield, IL) based on methods of Biermann and McGinnis (1989) and Churms et al. (1982). This method was based on the conversion of sucrose to fructose and glucose using enzyme Invertase ( $\beta$-fructosidase), and then the glucose produced measured by the hexokinase methods (Biermann and McGinnis, 1989). Sucrose measurement was based on dry matter basis (Wilcox and Shibles, 2001; Boydak et al., 2002; Bellaloui et al., 2010b).

\section{Seed Glucose and Fructose Analysis}

Glucose and fructose were determined in mature seed samples ground by a Laboratory Mill 3600 (Perten, Springfield, IL) to achieve particles uniformity. The Glucose Assay Reagent and sample blanks were prepared as recommended by the manufacturer and as detailed elsewhere by Bellaloui et al. (2014). The absorbance was read at $340 \mathrm{~nm}$ using a Beckman Coulter DU 800 spectrophotometer (Fullerton, CA). The concentration of glucose was expressed as $\mathrm{mg} \mathrm{g} \mathrm{dwt}-1$ and the concentration of fructose was measured as $\mathrm{mg} \mathrm{g} \mathrm{dwt}{ }^{-1}$.

\section{Total Boron and Seed Coat Boron Analysis}

Total and seed coat B were measured in mature seed at each harvesting times $[(0,14$, and 28 days after harvest maturity ( $\mathrm{H} 1$, $\mathrm{H} 2$, and $\mathrm{H} 3$ )]. In case of seed coat $\mathrm{B}$, the seeds were immersed in water for $12 \mathrm{~h}$, the seed coat was removed from the cotyledon, and the seed coat was dried in an oven for $16 \mathrm{~h}$ at $105^{\circ} \mathrm{C}$. Total and seed coat $\mathrm{B}$ were measured according to the AzomethineH method (Lohse, 1982), and as previously detailed elsewhere (Dordas, 2006; Dordas et al., 2007; Bellaloui et al., 2014). Briefly, the total $\mathrm{B}$ was measured in a 1.0-g ground seed sample. The sample was ashed at $500^{\circ} \mathrm{C}$, then extracted with $20 \mathrm{ml}$ of $2 \mathrm{M}$ $\mathrm{HCl}$ at $90^{\circ} \mathrm{C}$ for $10 \mathrm{~min}$, and then filtered. Then, a 2-ml sample of the solution was added to $4 \mathrm{ml}$ of buffer solution, containing $25 \%$ ammonium acetate, 1.5\% EDTA, and $12.5 \%$ acetic acid and $4 \mathrm{ml}$ of freshly prepared azomethine- $\mathrm{H}$ solution $(0.45 \%$ azomethine$\mathrm{H}$ and $1 \%$ of ascorbic acid) (John et al., 1975). After at least 45 min of color development, B concentration was determined in the samples using a Beckman Coulter DU 800 spectrophotometer (Fullerton, CA) at $420 \mathrm{~nm}$.

\section{Seed Coat Lignin}

The content of seed coat lignin of mature seed at different harvesting times was determined according to Alvarez et al. (1997) and Krzyzanowski et al. (2001). After the seeds were immersed in water for $12 \mathrm{~h}$, the seed coat was removed from the cotyledon, and the seed coat was dried in an oven for 16 $\mathrm{h}$ at $105^{\circ} \mathrm{C}$. Then, the samples were removed and placed in a desiccator at room temperature. Then, $250 \mathrm{mg}$ of dry seed coat were homogenized with a mortar and pestle in $7 \mathrm{ml}$ of $50 \mathrm{mM}$ potassium phosphate buffer at $\mathrm{pH} 7.0$ and the mixture was transferred into a centrifuge tube (Ferrarese et al., 2002) and centrifuged at $1,400 \mathrm{~g}$ for $10 \mathrm{~min}$. The pellet received three consecutive washes with different chemical solutions (twice with $7 \mathrm{ml}$ phosphate buffer $\mathrm{pH}$ 7.0; three times with $7 \mathrm{ml} \mathrm{1 \% (v/v)}$ Triton X-100 in pH 7.0 buffer; two times with $7 \mathrm{ml} 1 \mathrm{M} \mathrm{NaCl}$ in $\mathrm{pH} 7.0$ buffer; two times with $7 \mathrm{ml}$ with distilled water; and two times with $5 \mathrm{ml}$ acetone). The pellet was dried in an oven then placed into a vacuum desiccator to cool. This dry matter was considered as a protein-free cell wall fraction. A volume of $1.2 \mathrm{ml}$ thioglycolic acid with $6 \mathrm{ml} 2 \mathrm{M} \mathrm{HCl}$ was added to the dry matter and heated at $95^{\circ} \mathrm{C}$ for $4 \mathrm{~h}$. After cooling, the mixture was centrifuged at $1,400 \mathrm{~g}$ for $5 \mathrm{~min}$, and the supernatant was discarded. The pellet containing the lignin-thioglycolic acid (LTGA) complex was washed with distilled water, and the LTGA was extracted by shaking at $30^{\circ} \mathrm{C}$ for $18 \mathrm{~h}$ in $6 \mathrm{ml} 0.5 \mathrm{M} \mathrm{NaOH}$. After centrifugation at $1,400 \mathrm{~g}$ for $5 \mathrm{~min}$, the supernatant was stored and the pellet was washed again with $3 \mathrm{ml} 0.5 \mathrm{M} \mathrm{NaOH}$ and mixed with the supernatant. The combined alkali extracts were acidified with $1.8 \mathrm{ml}$ concentrated $\mathrm{HCl}$. After precipitation and LTGA recovery by cooling by cooling at $0^{\circ} \mathrm{C}$ for $4 \mathrm{~h}$ and centrifuging at 1,400 g for $5 \mathrm{~min}$ and washing two times with $7 \mathrm{ml}$ of distilled water, the pellet was then dried at $60^{\circ} \mathrm{C}$ and dissolved in $10 \mathrm{ml} 0.5 \mathrm{M} \mathrm{NaOH}$, and the insoluble material was removed by centrifugation. Lignin concentration was measured by reading the absorbance of the supernatant at $280 \mathrm{~nm}$ using a Beckman Coulter DU 800 spectrophotometer (Fullerton, CA). Lignin was expressed as mg LTGA/g dry weight.

\section{Experimental Design and Statistical Analysis}

The design of the experiment was completely randomized (CRD) with three replications. Analysis of variance was performed using PROC GLM in SAS (Statistical Analysis System, Copyright 20022010, Cary, NC, USA; Windows Version 6.1.7601). F and Pvalues are presented in Tables 2, 3. Level of significance was established as such ${ }^{*} P \leq 0.05 ;{ }^{* *} P \leq 0.01$; and ${ }^{* * *} P \leq 0.001$. Means were separated using Fisher's protected Least Significant Difference (LSD) (0.05). Level of significance was $\leq 0.05$ in all measured variables. PROC CORR in SAS was used to establish correlations ( $R$ and $P$-values) between variables.

\section{RESULTS}

\section{Analysis of Variance for Seed Composition and Seed Quality Components}

Analysis of variance showed that genotype $(G)$, harvest time $(\mathrm{H})$, and $\mathrm{G} \times \mathrm{Y} \times \mathrm{H}$ interactions were significant for seed protein and the fatty acids stearic, oleic, and linolenic (Table 2). Similar results for main effect factors and their interactions were observed for other seed composition and quality components. Protein, oil, sucrose, germination, accelerated aging (AA), and shattering were not affected by year, whereas all fatty acids, total and seed coat born, lignin, glucose, fructose, and hardseededness 
TABLE 2 | Analysis of variance ( $F$ and $P$-values) of the effects of year $(Y)$, genotype $(\mathrm{G})$, weathering [(delayed harvest $(\mathrm{H}) /$ time of harvest: harvest at harvest maturity (shortly after R8); 14 days after harvest maturity; and 28 days after harvest maturity)], and their interactions for seed protein, oil, fatty acids (\%), total boron (TB) (mg kg ${ }^{-1}$ ), seed coat boron (SCB) (mg kg $\left.{ }^{-1}\right)$, sugars [(sucrose; glucose; and fructose: ( $\left.\mathrm{mg} \mathrm{g}^{-1}\right)$ ], germination (Germ) (\%), accelerated aging (AA) \%, hard-seed (HS) (\%), and shattering (Shat) (\%) in soybean breeding lines differing in seed germinability under high heat conditions.

\begin{tabular}{|c|c|c|c|c|c|c|c|c|c|c|c|c|c|}
\hline \multirow[b]{2}{*}{ Effect } & \multirow[b]{2}{*}{ DF } & \multicolumn{2}{|c|}{ Protein } & \multicolumn{2}{|c|}{ Oil } & \multicolumn{2}{|c|}{ Palmitic } & \multicolumn{2}{|c|}{ Stearic } & \multicolumn{2}{|c|}{ Oleic } & \multicolumn{2}{|c|}{ Linolenic } \\
\hline & & $\boldsymbol{F}$ & $\boldsymbol{P}$ & $\boldsymbol{F}$ & $\boldsymbol{P}$ & $\boldsymbol{F}$ & $\boldsymbol{P}$ & $\boldsymbol{F}$ & $P$ & $\boldsymbol{F}$ & $P$ & $\boldsymbol{F}$ & $P$ \\
\hline Year $(Y)$ & 1 & 1.5 & NS & 10.3 & $\star \star \star$ & 1,159 & 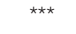 & 260 & 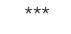 & 98 & 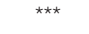 & 410 & $\star \star * \star$ \\
\hline Genotype (G) & 11 & 78 &  & 42.6 & 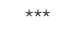 & 13.0 & 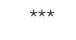 & 2.3 & $\star \star$ & 28 & 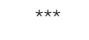 & 16.9 & 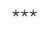 \\
\hline Harvest (H) & 2 & 13.2 & $\star * \star \star$ & 12.8 &  & 0.19 & NS & 11.1 & 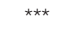 & 128 & 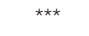 & 29.8 & $* \star *$ \\
\hline$Y \times G$ & 11 & 32.8 & $* * *$ & 20.9 & 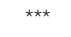 & 2.3 & ** & 3.6 &  & 17.5 & 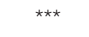 & 20.6 & $* \star \star *$ \\
\hline $\mathrm{Y} \times \mathrm{H}$ & 2 & 6.1 & 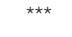 & 1.3 & NS & 0.64 & NS & 0.36 & NS & 31.1 & $\star \star \star$ & 24.9 & $\star \star \star *$ \\
\hline $\mathrm{G} \times \mathrm{H}$ & 22 & 4.6 & 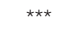 & 4.6 & 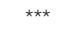 & 1.6 & * & 0.63 & NS & 3.3 & $\star \star \star$ & 7.4 & 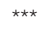 \\
\hline $\mathrm{Y} \times \mathrm{G} \times \mathrm{H}$ & 21 & 3.7 &  & 7.6 & 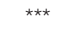 & 2.0 & ** & 0.5 & NS & 8.3 & $\star \star \star \star ~$ & 6.4 & $* \star *$ \\
\hline \multicolumn{14}{|c|}{ SEED QUALITY COMPONENT } \\
\hline & & \multicolumn{2}{|c|}{ TotB } & \multicolumn{2}{|c|}{ SCB } & \multicolumn{2}{|c|}{ Lignin } & \multicolumn{2}{|c|}{ Sucrose } & \multicolumn{2}{|c|}{ Glucose } & & \\
\hline Effect & DF & $\boldsymbol{F}$ & $\boldsymbol{P}$ & $\boldsymbol{F}$ & $\boldsymbol{P}$ & $\boldsymbol{F}$ & $\boldsymbol{P}$ & $\boldsymbol{F}$ & $\boldsymbol{P}$ & $\boldsymbol{F}$ & $\boldsymbol{P}$ & & \\
\hline Year $(Y)$ & 1 & 416 & 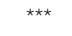 & 140 & $* \star *$ & 159 & $\star \star \star \star *$ & 344 & $\star \star \star *$ & 9.9 & 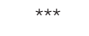 & & \\
\hline Genotype (G) & 11 & 41.8 & *** & 66.8 & $* \star \star *$ & 36.0 & $\star * \star \star$ & 7.9 & 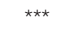 & 3.3 & 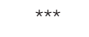 & & \\
\hline Harvest $(\mathrm{H})$ & 2 & 926 & $\star \star \star \star$ & 234 & $\star \star \star ~$ & 8.1 & 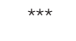 & 125 & 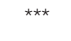 & 313 &  & & \\
\hline$Y \times G$ & 11 & 11.6 & 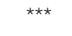 & 2.7 & 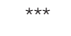 & 2.3 & ** & 1.7 & NS & 0.84 & NS & & \\
\hline $\mathrm{Y} \times \mathrm{H}$ & 2 & 22.8 & 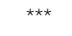 & 0.68 & NS & 3.5 & * & 3.7 & * & 28.1 &  & & \\
\hline $\mathrm{G} \times \mathrm{H}$ & 22 & 6.7 &  & 8.8 & 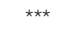 & 1.3 & NS & 3.3 & $\star \star \star ~$ & 4.8 & 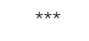 & & \\
\hline $\mathrm{Y} \times \mathrm{G} \times \mathrm{H}$ & 21 & 8.1 & 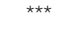 & 7.9 &  & 1.8 & * & 1.2 & NS & 2.2 &  & & \\
\hline \multicolumn{14}{|c|}{ SEED QUALITY COMPONENT } \\
\hline & & \multicolumn{2}{|c|}{ Fructose } & \multicolumn{2}{|c|}{ Germ } & \multicolumn{2}{|c|}{ AA } & \multicolumn{2}{|c|}{ HS } & \multicolumn{2}{|c|}{ Shat } & & \\
\hline Effect & DF & $\boldsymbol{F}$ & $\boldsymbol{P}$ & $\boldsymbol{F}$ & $\boldsymbol{P}$ & $\boldsymbol{F}$ & $\boldsymbol{P}$ & $\boldsymbol{F}$ & $\boldsymbol{P}$ & $\boldsymbol{F}$ & $\boldsymbol{P}$ & & \\
\hline Year (Y) & 128 & 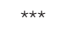 & 28.2 & 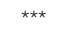 & 120 & 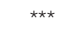 & 32.8 & 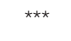 & 1.3 & NS & 127.7 & & \\
\hline Genotype (G) & 6.7 & 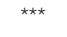 & 20.9 & 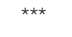 & 21.9 &  & 28.5 &  & 19.6 & 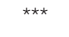 & 6.7 & & \\
\hline Harvest $(\mathrm{H})$ & 123 &  & 119 & 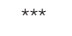 & 233 &  & 0.84 & NS & 76.3 & $\star \star \star *$ & 123 & & \\
\hline$Y \times G$ & 4.3 & $\star \star \star$ & 12.3 & 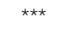 & 2.1 & * & 32.2 & 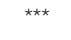 & 11.4 &  & 4.3 & & \\
\hline $\mathrm{Y} \times \mathrm{H}$ & 11.0 & $\star \star \star$ & 2.6 & NS & 7.7 & $\star \star \star \star ~$ & 0.37 & NS & 12.3 & $\star \star \star ~$ & 11.0 & & \\
\hline $\mathrm{G} \times \mathrm{H}$ & 3.3 & $\star \star \star$ & 2.0 &  & 4.5 & $\star \star \star \star$ & 4.8 & 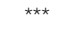 & 7.7 & 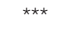 & 3.3 & & \\
\hline $\mathrm{Y} \times \mathrm{G} \times \mathrm{H}$ & 2.5 & $\star \star \star *$ & 4.3 & $* \star \star *$ & 6.9 & 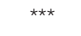 & 3.8 & 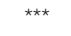 & 3.7 & $\star \star \star *$ & 2.5 & & \\
\hline
\end{tabular}

Plants were grown in a Sharkey clay soil in 2010 and 2011 at Stoneville, MS, USA.

${ }^{\star} P \leq 0.05 ;{ }^{* \star} P \leq 0.01 ;{ }^{* \star} P \leq 0.001 ; N S$, not significant; $D F$, degree of freedom.

(HS) were all affected by year. In addition, genotypic background (GB) (exotic lines vs non-exotic checks) had significant effects on all seed composition components, except stearic and linolenic acids and glucose (Table 3 ). There were no significant effects of delayed harvest $(\mathrm{H})$ on some seed composition, such as protein, oil, and palmitic and stearic acids when exotic lines vs non-exotic checks were compared. The interaction between GB and Y was significant for seed composition components, except linolenic acid, SCB, sucrose, and glucose. So, GB $\times$ Y interactions had significant effects for the seed composition components protein, oil, palmitic acid, stearic acid, oleic acid, linoleic acid, total boron, lignin, and fructose (Table 3). Except for palmitic acid the $\mathrm{Y} \times \mathrm{GB} \times \mathrm{H}$ interactions were not significant for seed composition components. For $\mathrm{GB} \times \mathrm{H}$ interactions, only $\mathrm{SCB}$ and glucose were significant (Table 3 ). Genotypic background also had significant effects on total boron (TB) and seed coat $\mathrm{B}$ (SCB), sucrose, fructose, and seed quality (germination, hard seed, and accelerated aging) (Table 3 ). Since the interactions between the main effect factors $(\mathrm{G} \times \mathrm{H} \times \mathrm{Y})$ were significant for many seed composition components (Table 2), the results will be presented separately by year as done previously by Bellaloui et al. (2017).

\section{Effects of Delayed Harvest on Seed Protein, Fatty Acids, Total B and Seed Coat B}

Seed protein and oleic acid were higher in the breeding lines with HG (34-3-1-2-4-1, 30-1-4-1-1, 25-1-1-1-1-4, and 24-2-1-2$1-2)$ than in the non-exotic checks for the non-delayed harvest 
TABLE 3 | Analysis of variance ( $F$ and $P$-values) of the effects of year $(Y)$, genotypic background (GB) [(two genotypic sources were used: exotic lines and parents and non-exotic (parents and commercial cultivars)], and weathering [(delayed harvest ( $\mathrm{H}) /$ time of harvest: harvest at harvest maturity (shortly after R8); 14 days after harvest maturity; and 28 days after harvest maturity)], and their interactions for seed protein, oil, fatty acids (\%), total boron (TB) (mg kg $\left.{ }^{-1}\right)$, seed coat boron (SCB) (mg kg ${ }^{-1}$ ), sugars [(sucrose, glucose, and fructose: $\left(\mathrm{mg} \mathrm{g}^{-1}\right)$ ], germination (Germ) (\%), accelerated aging (AA)\%, hard-seed (HS) (\%), and shattering (Shat) (\%) in soybean breeding lines differing in seed germinability under high heat conditions.

\begin{tabular}{|c|c|c|c|c|c|c|c|c|c|c|c|c|c|c|c|}
\hline \multirow[b]{2}{*}{ Effect } & \multirow[b]{2}{*}{ DF } & \multicolumn{2}{|c|}{ Protein } & \multicolumn{2}{|c|}{ Oil } & \multicolumn{2}{|c|}{ Palmitic } & \multicolumn{2}{|c|}{ Stearic } & \multicolumn{2}{|c|}{ Oleic } & \multicolumn{2}{|c|}{ Linoleic } & \multicolumn{2}{|c|}{ Linolenic } \\
\hline & & $F$ & $P$ & $F$ & $P$ & $\boldsymbol{F}$ & $P$ & $\boldsymbol{F}$ & $P$ & $\boldsymbol{F}$ & $\boldsymbol{P}$ & $\boldsymbol{F}$ & $P$ & $\boldsymbol{F}$ & $P$ \\
\hline Year $(Y)$ & 1 & 0.76 & NS & 4.4 & * & 886 & $\star \star \star ~$ & 237 &  & 32 & 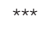 & 62 & $\star \star \star \star$ & 92 & $\star \star \star \star ~$ \\
\hline Genotypic Background (GB) & 1 & 9.6 &  & 4.3 & * & 6.8 & * & 4.8 & * & 9.8 & $\star \star \star$ & 19.7 &  & 0.76 & NS \\
\hline Harvest(H) & 2 & 0.94 & NS & 0.31 & NS & 0.93 & NS & 0.52 & NS & 11.2 & 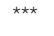 & 19.5 & $\star \star \star *$ & 3.9 & * \\
\hline$Y \times G B$ & 1 & 9.6 & 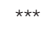 & 4.3 & * & 6.8 & * & 4.8 & * & 9.7 & 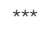 & 19.7 & $\star \star \star ~$ & 0.76 & NS \\
\hline $\mathrm{Y} \times \mathrm{H}$ & 2 & 0.94 & NS & 0.31 & NS & 0.93 & NS & 0.52 & NS & 11.2 & 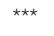 & 19.5 &  & 3.9 & * \\
\hline $\mathrm{GB} \times \mathrm{H}$ & 2 & 0.15 & NS & 0.3 & NS & 3.8 & * & 0.68 & NS & 0.54 & NS & 2.2 & NS & 0.79 & NS \\
\hline $\mathrm{Y} \times \mathrm{GB} \times \mathrm{H}$ & 2 & 0.15 & NS & 0.3 & NS & 3.8 & * & 0.68 & NS & 0.54 & NS & 2.2 & NS & 0.79 & NS \\
\hline \multicolumn{16}{|c|}{ SEED QUALITY COMPONENT } \\
\hline & & \multicolumn{2}{|c|}{ TB } & \multicolumn{2}{|c|}{ SCB } & \multicolumn{2}{|c|}{ Lignin } & \multicolumn{2}{|c|}{ Sucrose } & \multicolumn{2}{|c|}{ Glucose } & & & & \\
\hline Effect & DF & $\boldsymbol{F}$ & $P$ & $\boldsymbol{F}$ & $P$ & $F$ & $P$ & $\boldsymbol{F}$ & $\boldsymbol{P}$ & $\boldsymbol{F}$ & $\boldsymbol{P}$ & & & & \\
\hline Year $(Y)$ & 1 & 113 & $\star \star \star \star ~$ & 41 & 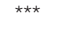 & 77 & $\star \star \star ~$ & 236 & 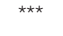 & 7.7 & 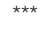 & & & & \\
\hline Genotypic Background(GB) & 1 & 9.4 & 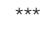 & 1.3 & NS & 4.9 & * & 1.2 & NS & 2.5 & NS & & & & \\
\hline Harvest(H) & 2 & 5.9 & 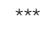 & 0.12 & NS & 1.7 & NS & 2.5 & NS & 20.2 & $\star \star \star$ & & & & \\
\hline $\mathrm{Y} \times \mathrm{GB}$ & 1 & 9.4 &  & 1.3 & NS & 4.9 & * & 1.2 & NS & 2.5 & NS & & & & \\
\hline $\mathrm{Y} \times \mathrm{H}$ & 2 & 5.9 &  & 0.12 & NS & 1.7 & NS & 2.5 & NS & 20.2 & $\star \star \star$ & & & & \\
\hline $\mathrm{GB} \times \mathrm{H}$ & 2 & 1.4 & NS & 0.44 & NS & 0.59 & NS & 0.94 & NS & 4.1 & * & & & & \\
\hline $\mathrm{Y} \times \mathrm{GB} \times \mathrm{H}$ & 2 & 1.4 & NS & 0.44 & NS & 0.59 & NS & 0.94 & NS & 4.1 & * & & & & \\
\hline \multicolumn{16}{|c|}{ SEED QUALITY COMPONENT } \\
\hline & & \multicolumn{2}{|c|}{ Fructose } & \multicolumn{2}{|c|}{ Germ } & \multicolumn{2}{|c|}{ AA } & \multicolumn{2}{|c|}{ HS } & \multicolumn{2}{|c|}{ Shat } & & & & \\
\hline Effect & DF & $\boldsymbol{F}$ & $P$ & $\boldsymbol{F}$ & $P$ & $\boldsymbol{F}$ & $P$ & $\boldsymbol{F}$ & $P$ & $\boldsymbol{F}$ & $P$ & & & & \\
\hline Year $(Y)$ & 1 & 78 &  & 11.4 &  & 56 & 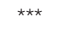 & 8.1 &  & 0.65 & NS & & & & \\
\hline Genotypic Background(GB) & 1 & 11.6 & 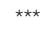 & 7.6 &  & 1.4 & NS & 3.5 & NS & 1.3 & NS & & & & \\
\hline Harvest(H) & 2 & 6.7 & 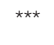 & 0.84 & NS & 3.4 & * & 0.17 & NS & 3.9 & * & & & & \\
\hline$Y \times G B$ & 1 & 11.6 &  & 7.6 & 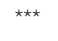 & 1.4 & NS & 3.5 & NS & 1.3 & NS & & & & \\
\hline $\mathrm{Y} \times \mathrm{H}$ & 2 & 6.7 & $\star \star \star$ & 0.84 & NS & 3.4 & * & 0.17 & NS & 3.9 & $\star$ & & & & \\
\hline $\mathrm{GB} \times \mathrm{H}$ & 2 & 2.5 & NS & 0.87 & NS & 3.0 & * & 0.18 & NS & 0.71 & NS & & & & \\
\hline $\mathrm{Y} \times \mathrm{GB} \times \mathrm{H}$ & 2 & 2.5 & NS & 0.87 & NS & 3.0 & * & 0.18 & NS & 0.72 & NS & & & & \\
\hline
\end{tabular}

Plants were grown in a Sharkey clay soil in 2010 and 2011 at Stoneville, MS, USA.

${ }^{\star} P \leq 0.05 ;{ }^{* \star} P \leq 0.01 ;{ }^{* \star} P \leq 0.001 ; N S$, not significant; $D F$, degree of freedom.

(H1) in 2010 and 2011 (Tables 4, 5). The HG lines showed different levels of seed composition components in 2010 and 2011(Tables 4, 5). Harvest time showed effects on oleic acid, as it declined for all lines, except LD00-3309 between $\mathrm{H} 2$ and H3, after delayed harvests, especially at $\mathrm{H} 3$ (28 days after initial harvest). Seed protein decline was observed in only some genotypes. Total B (TB) and seed coat B (SCB) were higher in the HG breeding lines (34-3-1-2-4-1, 30-1-4-1-1, 25-1-1-1-1-4, and 242-1-2-1-2) compared to the non-exotic checks, and all parents (PI587982A, DT98-9102, DT97-4290, PI603756, and 5601T) for the H1 harvest in 2010 and 2011 (Tables 4, 5). Generally, TB and SCB decreased in all genotypes after delays in harvest, especially after 28 days of delayed harvest (H3). However, the level of SCB in $\mathrm{HG}$ lines was higher after $\mathrm{H} 3$ compared with the non-exotic checks (Tables 4,5 ).

\section{Effects of Delayed Harvest on Seed Coat Lignin and Sugars}

Lignin was higher in the HG breeding lines (34-3-1-2-4-1, 30-1-4-1-1, 25-1-1-1-1-4, and 24-2-1-2-1-2) compared to the non-exotic checks for all three harvests (H1, H2, and H3) in 2010 and 2011 (Tables 4, 5). Generally, lignin decreased after delays in harvest (H2 and $\mathrm{H} 3$ ) in all genotypes, but the level of lignin content in the HG breeding lines was still higher at each harvest time compared to the non-exotic checks. Levels of sucrose and glucose generally declined with delays in harvest for most lines, whereas fructose levels were similar across times of harvest. Seed sucrose and glucose showed inconsistent differences between HG lines and non-exotic checks across harvest times and years. Breeding lines and other genotypes accumulated different levels of lignin and sugars (Tables 4, 5). A similar pattern 
TABLE 4 | Effect of weathering [(delayed harvest $(\mathrm{H}) /$ time of harvest: harvest at harvest maturity, shortly after R8 (H1); 14 days after harvest maturity (H2); and 28 days after harvest maturity (H3)] on seed protein (P), oil (O), fatty acids stearic (St), oleic (OI), linoleic (lin), and linolenic (lino) (\%), total boron (TB) (mg kg $\left.{ }^{-1}\right)$, seed coat boron

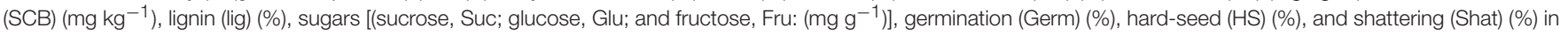
soybean breeding lines differing in seed germinability under high heat conditions.

\begin{tabular}{|c|c|c|c|c|c|c|c|c|c|c|c|c|c|c|c|c|c|c|}
\hline Genotype & $\mathbf{P}$ & $\mathbf{P}$ & $\mathbf{P}$ & 0 & 0 & 0 & St & St & St & OI & OI & OI & Lin & Lin & Lin & Lino & Lino & Lino \\
\hline HG (Exotic) & H1 & $\mathrm{H} 2$ & H3 & H1 & $\mathrm{H} 2$ & H3 & H1 & H2 & H3 & H1 & H2 & H3 & H1 & H2 & H3 & H1 & $\mathrm{H} 2$ & H3 \\
\hline $34-3-1-2-4-1$ & 44.6 & 44.3 & 43.3 & 18.9 & 19.2 & 18.6 & 3.5 & 3.2 & 3.1 & 28.9 & 25.7 & 24.2 & 50.1 & 52.7 & 54.6 & 5.6 & 5.2 & 5.5 \\
\hline $30-1-4-1-1$ & 43.3 & 43.7 & 43.1 & 19.1 & 18.8 & 18.9 & 2.8 & 2.8 & 2.8 & 29.3 & 25.1 & 23.0 & 49.2 & 51.6 & 54.2 & 6.6 & 6.8 & 6.6 \\
\hline $25-1-1-1-1-4$ & 42.8 & 42.9 & 42.5 & 19.7 & 19.4 & 18.7 & 3.2 & 2.8 & 2.9 & 27.8 & 25.1 & 23.2 & 51.1 & 53.4 & 54.5 & 5.6 & 5.7 & 6.1 \\
\hline $24-2-1-2-1-2$ & 43.9 & 42.8 & 41.3 & 20.2 & 20.3 & 22.3 & 3.2 & 3.0 & 3.3 & 28.4 & 24.1 & 22.4 & 51.8 & 54.4 & 55.1 & 5.7 & 5.9 & 5.6 \\
\hline PI603756 & 43.8 & 40.7 & 42.1 & 19.1 & 20.6 & 22.3 & 3.2 & 3.0 & 3.4 & 30.1 & 25.9 & 23.1 & 47.4 & 53.5 & 55.5 & 5.5 & 5.9 & 5.8 \\
\hline PI587982A & 41.4 & 40.4 & 41.3 & 22.8 & 21.1 & 21.8 & 3.5 & 3.4 & 3.3 & 26.0 & 24.8 & 21.6 & 52.1 & 52.7 & 57.0 & 5.8 & 5.9 & 6.1 \\
\hline \multicolumn{19}{|l|}{ CHECKS } \\
\hline LD00-3309 & 42.4 & 42.7 & 42.2 & 19.0 & 20.1 & 21.8 & 3.1 & 2.6 & 2.6 & 22.5 & 20.7 & 21.7 & 56.7 & 57.5 & 57.0 & 6.1 & 6.4 & 6.3 \\
\hline DT98-9102 & 40.9 & 40.9 & 41.2 & 20.6 & 21.2 & 20.5 & 3.5 & 3.1 & 3.0 & 25.7 & 23.9 & 21.9 & 54.3 & 55.8 & 56.8 & 5.6 & 5.6 & 5.5 \\
\hline DT97-4290 & 42.0 & 40.6 & 41.2 & 19.8 & 21.5 & 20.2 & 3.0 & 2.9 & 2.8 & 27.5 & 23.1 & 22.1 & 51.1 & 55.4 & 56.3 & 5.2 & 6.1 & 5.8 \\
\hline AG4903 & 40.1 & 40.7 & 40.8 & 21.7 & 21.8 & 21.2 & 3.2 & 3.0 & 3.0 & 27.6 & 22.8 & 20.1 & 52.2 & 57.5 & 58.1 & 5.5 & 5.4 & 5.4 \\
\hline 94B73 & 41.0 & 40.6 & 40.6 & 20.5 & 21.4 & 22.1 & 3.5 & 3.1 & 3.1 & 23.7 & 22.7 & 21.0 & 55.8 & 55.3 & 58.4 & 5.3 & 5.8 & 5.5 \\
\hline $5601 T$ & 41.2 & 40.6 & 41.3 & 20.4 & 21.1 & 20.4 & 3.1 & 2.8 & 2.9 & 23.1 & 23.0 & 18.0 & 56.1 & 56.0 & 60.1 & 5.8 & 6.3 & 5.8 \\
\hline LSD & 0.40 & 0.54 & 0.38 & 0.40 & 0.57 & 0.40 & 0.20 & 0.23 & 0.20 & 0.70 & 0.60 & 0.76 & 1.10 & 0.92 & 0.82 & 0.30 & 0.41 & 0.40 \\
\hline \multicolumn{19}{|c|}{ SEED QUALITY COMPONENT } \\
\hline Genotype & TB & TB & TB & SCB & SCB & SCB & Lig & Lig & Lig & Suc & Suc & Suc & Glu & Glu & Glu & Fruc & Fruc & Fruc \\
\hline HG (Exotic) & H1 & $\mathrm{H} 2$ & H3 & H1 & H2 & H3 & H1 & H2 & H3 & H1 & H2 & H3 & H1 & $\mathrm{H} 2$ & H3 & H1 & H2 & H3 \\
\hline $34-3-1-2-4-1$ & 43.8 & 37.8 & 28.9 & 31.1 & 28.6 & 24.7 & 5.6 & 5.8 & 4.7 & 49.3 & 45.7 & 44.0 & 3.8 & 3.9 & 3.2 & 0.8 & 0.9 & 0.8 \\
\hline $30-1-4-1-1$ & 42.6 & 39.0 & 29.5 & 29.8 & 29.3 & 24.8 & 6.0 & 5.1 & 5.1 & 45.6 & 45.3 & 43.3 & 3.8 & 3.8 & 3.5 & 0.9 & 1.0 & 0.8 \\
\hline $25-1-1-1-1-4$ & 42.3 & 38.6 & 34.1 & 30.4 & 31.2 & 26.4 & 4.5 & 5.2 & 5.3 & 45.8 & 43.0 & 43.4 & 4.3 & 3.9 & 3.1 & 1.0 & 0.9 & 0.7 \\
\hline $24-2-1-2-1-2$ & 42.2 & 40.0 & 31.2 & 29.7 & 30.9 & 26.7 & 6.0 & 5.5 & 5.5 & 50.8 & 43.7 & 42.2 & 4.0 & 4.5 & 3.3 & 1.1 & 1.2 & 0.8 \\
\hline PI603756 & 40.0 & 37.6 & 29.2 & 28.9 & 26.6 & 23.9 & 4.8 & 4.2 & 3.4 & 44.7 & 43.3 & 32.8 & 3.8 & 3.9 & 2.7 & 0.9 & 1.0 & 0.6 \\
\hline PI587982A & 40.5 & 38.6 & 30.3 & 28.3 & 26.5 & 23.8 & 4.4 & 3.9 & 3.5 & 45.9 & 40.8 & 33.7 & 3.5 & 4.5 & 2.4 & 0.9 & 1.0 & 0.6 \\
\hline \multicolumn{19}{|l|}{ CHECKS } \\
\hline LD00-3309 & 39.6 & 35.2 & 28.9 & 21.8 & 26.9 & 21.9 & 3.0 & 3.6 & 3.5 & 45.3 & 44.8 & 33.3 & 4.0 & 4.4 & 2.5 & 0.9 & 0.9 & 0.6 \\
\hline DT98-9102 & 40.5 & 35.2 & 29.8 & 27.0 & 26.7 & 22.3 & 3.7 & 3.7 & 3.4 & 44.1 & 45.3 & 34.4 & 3.8 & 4.2 & 2.5 & 1.1 & 0.9 & 0.6 \\
\hline DT97-4290 & 41.9 & 36.8 & 27.7 & 26.9 & 24.3 & 21.6 & 3.7 & 4.1 & 3.6 & 47.0 & 45.8 & 34.9 & 3.8 & 4.7 & 2.2 & 0.9 & 1.2 & 0.7 \\
\hline AG4903 & 41.2 & 36.5 & 30.0 & 22.2 & 27.3 & 21.1 & 3.5 & 3.6 & 3.3 & 46.2 & 46.5 & 33.3 & 3.7 & 4.1 & 2.3 & 1.0 & 1.0 & 0.6 \\
\hline $94 \mathrm{~B} 73$ & 39.9 & 35.1 & 28.5 & 25.5 & 25.0 & 19.9 & 3.4 & 3.9 & 3.1 & 43.9 & 44.6 & 35.5 & 3.8 & 3.9 & 2.5 & 0.6 & 1.0 & 0.6 \\
\hline $5601 \mathrm{~T}$ & 37.5 & 33.2 & 27.9 & 20.6 & 26.4 & 20.4 & 3.2 & 3.4 & 3.8 & 43.8 & 41.3 & 34.7 & 4.4 & 3.7 & 2.4 & 0.9 & 1.1 & 0.6 \\
\hline LSD & 0.60 & 0.65 & 1.11 & 0.70 & 0.71 & 0.76 & 0.3 & 0.26 & 0.30 & 1.30 & 0.91 & 0.69 & 2.00 & 0.23 & 0.21 & 0.10 & 0.06 & 0.03 \\
\hline \multicolumn{19}{|c|}{ SEED QUALITY COMPONENT } \\
\hline Genotype & Germ & Germ & Germ & AA & AA & AA & HS & HS & HS & & & & & & & & & \\
\hline HG (Exotic) & H1 & H2 & H3 & H1 & H2 & H3 & H1 & H2 & H3 & & & & & & & & & \\
\hline $34-3-1-2-4-1$ & 92.7 & 71.3 & 74.0 & 66.0 & 32.7 & 52.7 & 0.0 & 0.0 & 0.7 & & & & & & & & & \\
\hline $30-1-4-1-1$ & 96.0 & 93.7 & 67.3 & 83.0 & 71.0 & 14.0 & 0.3 & 0.0 & 0.0 & & & & & & & & & \\
\hline $25-1-1-1-1-4$ & 92.7 & 72.3 & 72.0 & 73.7 & 39.3 & 26.7 & 0.0 & 0.7 & 8.3 & & & & & & & & & \\
\hline $24-2-1-2-1-2$ & 83.7 & 64.0 & 65.0 & 68.0 & 25.7 & 16.0 & 0.0 & 0.3 & 8.0 & & & & & & & & & \\
\hline PI603756 & 86.0 & 74.3 & 48.0 & 93.0 & 82.0 & 3.3 & 5.3 & 11.7 & 0.0 & & & & & & & & & \\
\hline PI587982A & 95.7 & 68.3 & 32.0 & 87.0 & 17.7 & 2.3 & 0.3 & 0.0 & 0.0 & & & & & & & & & \\
\hline \multicolumn{19}{|l|}{ CHECKS } \\
\hline LD00-3309 & 37.3 & 23.0 & 11.0 & 25.0 & 21.7 & 19.0 & 29.3 & 22.3 & 25.0 & & & & & & & & & \\
\hline DT98-9102 & 88.7 & 83.3 & 74.3 & 48.7 & 29.0 & 37.0 & 0.3 & 1.0 & 1.3 & & & & & & & & & \\
\hline DT97-4290 & 40.7 & 12.0 & 31.7 & 31.7 & 10.3 & 11.7 & 1.0 & 1.3 & 1.3 & & & & & & & & & \\
\hline AG4903 & 44.0 & 39.0 & 33.3 & 36.7 & 16.7 & 18.7 & 10.7 & 9.7 & 20.3 & & & & & & & & & \\
\hline $94 \mathrm{~B} 73$ & 56.7 & 45.3 & 22.7 & 34.3 & 25.7 & 6.0 & 17.7 & 12.7 & 0.7 & & & & & & & & & \\
\hline $5601 \mathrm{~T}$ & 75.7 & 51.7 & 55.7 & 40.3 & 25.3 & 17.3 & 0.7 & 2.3 & 0.3 & & & & & & & & & \\
\hline LSD & 3.70 & 4.19 & 8.75 & 3.90 & 4.07 & 7.75 & 1.50 & 0.79 & 2.30 & & & & & & & & & \\
\hline
\end{tabular}

Plants were grown in a Sharkey clay soil in 2010 at Stoneville, MS, USA. 
TABLE 5 | Effect of weathering [(delayed harvest $(\mathrm{H}) /$ time of harvest: harvest at harvest maturity, shortly after R8 (H1); 14 days after harvest maturity (H2); and 28 days after harvest maturity ( $\mathrm{H3})$ ] on seed protein $(\mathrm{P})$, oil $(\mathrm{O})$, fatty acids stearic (St), oleic (OI), linoleic (lin), and linolenic (lino) (\%),total boron (TB) (mg kg $\left.{ }^{-1}\right)$, cell wall boron (CWB) (mg kg $\left.{ }^{-1}\right)$, lignin (lig) (\%), sugars [(sucrose, Suc; glucose, Glu; and fructose, Fru: $\left(\mathrm{mg} \mathrm{g}^{-1}\right)$ ], germination (Germ) (\%), accelerating aging (AA) (\%), hard-seed (HS) (\%), and shattering (Shat) (\%) in soybean breeding lines differing in seed germinability under high heat conditions.

\begin{tabular}{|c|c|c|c|c|c|c|c|c|c|c|c|c|c|c|c|c|c|c|}
\hline & & $\mathbf{P}$ & & & 0 & & & St & & & OI & & & Lin & & & Lino & \\
\hline HG (Exotic) & H1 & $\mathrm{H} 2$ & Н3 & H1 & $\mathrm{H} 2$ & H3 & H1 & H2 & H3 & H1 & H2 & H3 & H1 & H2 & H3 & H1 & H2 & H3 \\
\hline $34-3-1-2-4-1$ & 43.0 & 43.5 & 40.8 & 19.9 & 20.0 & 21.2 & 4.1 & 4.1 & 3.9 & 29.5 & 31.0 & 24.1 & 50.3 & 48.9 & 53.0 & 6.4 & 5.9 & 8.4 \\
\hline $30-1-4-1-1$ & 44.8 & 45.7 & 43.4 & 18.0 & 17.9 & 20.0 & 3.8 & 3.6 & 3.9 & 27.3 & 29.2 & 30.7 & 52.0 & 51.0 & 49.2 & 5.3 & 5.3 & 6.3 \\
\hline $25-1-1-1-1-4$ & 45.5 & 45.8 & 44.0 & 17.9 & 17.8 & 17.6 & 3.8 & 3.7 & 3.5 & 26.5 & 27.1 & 25.1 & 52.8 & 51.5 & 51.7 & 5.7 & 6.2 & 8.5 \\
\hline $24-2-1-2-1-2$ & 45.9 & 46.3 & 46.9 & 18.0 & 17.8 & 17.4 & 3.8 & 3.6 & 3.4 & 29.0 & 30.7 & 26.3 & 50.7 & 50.3 & 51.1 & 6.2 & 5.5 & 8.4 \\
\hline PI603756 & 41.5 & 40.2 & . & 21.5 & 21.6 & . & 4.0 & 3.5 & . & 20.0 & 21.5 & . & 55.6 & 53.6 & . & 10.2 & 10.9 & . \\
\hline PI587982A & 41.9 & 40.7 & 40.6 & 20.0 & 20.0 & 21.7 & 3.6 & 3.5 & 3.5 & 27.5 & 20.4 & 21.6 & 51.7 & 55.5 & 54.2 & 5.8 & 10.2 & 9.8 \\
\hline \multicolumn{19}{|l|}{ CHECKS } \\
\hline LD00-3309 & 40.4 & 40.0 & 40.8 & 20.1 & 20.7 & 20.1 & 4.0 & 3.8 & 3.5 & 24.7 & 26.9 & 20.9 & 52.1 & 52.4 & 53.8 & 6.4 & 6.9 & 10.6 \\
\hline DT98-9102 & 40.0 & 40.7 & 39.3 & 21.1 & 21.2 & 20.7 & 3.8 & 3.8 & 3.8 & 25.2 & 25.0 & 26.7 & 54.2 & 53.7 & 52.6 & 8.9 & 9.1 & 7.0 \\
\hline DT97-4290 & 40.3 & 40.4 & 40.5 & 21.5 & 21.8 & 21.1 & 4.1 & 4.0 & 3.7 & 25.5 & 25.1 & 24.0 & 50.8 & 52.4 & 54.2 & 5.6 & 6.2 & 8.7 \\
\hline AG4903 & 43.7 & 44.1 & 40.2 & 19.0 & 18.9 & 21.9 & 3.7 & 3.5 & 3.9 & 25.7 & 26.9 & 27.2 & 52.2 & 52.9 & 54.1 & 7.6 & 6.3 & 5.6 \\
\hline $94 \mathrm{~B} 73$ & 41.0 & 40.6 & 42.1 & 21.9 & 22.1 & 18.8 & 3.9 & 3.8 & 3.5 & 26.4 & 26.4 & 24.4 & 53.3 & 52.6 & 52.9 & 6.2 & 7.4 & 8.3 \\
\hline $5601 \mathrm{~T}$ & 39.5 & 39.9 & 40.8 & 20.7 & 21.2 & 22.4 & 4.0 & 3.9 & 3.7 & 23.9 & 24.4 & 25.7 & 53.0 & 53.1 & 53.1 & 9.2 & 8.0 & 8.0 \\
\hline LSD & 0.37 & 0.30 & 0.34 & 0.17 & 0.20 & 0.18 & 0.14 & 0.06 & 0.059 & 0.77 & 0.62 & 0.66 & 0.59 & 0.60 & 0.6 & 0.27 & 0.28 & 0.36 \\
\hline \multicolumn{19}{|c|}{ SEED QUALITY COMPONENT } \\
\hline Genotype & TB & TB & TB & СВ & СB & СВ & Lig & Lig & Lig & Suc & Suc & Suc & Glu & Glu & Glu & Fru & Fru & Fru \\
\hline HG (Exotic) & H1 & $\mathrm{H} 2$ & Н3 & H1 & $\mathrm{H} 2$ & H3 & H1 & H2 & H3 & H1 & $\mathrm{H} 2$ & H3 & H1 & $\mathrm{H} 2$ & H3 & H1 & H2 & H3 \\
\hline $34-3-1-2-4-1$ & 48.5 & 41.4 & 36.1 & 34.6 & 31.8 & 26.9 & 4.7 & 3.9 & 4.5 & 61.6 & 53.5 & 43.2 & 4.0 & 4.0 & 3.5 & 1.2 & 1.2 & 1.0 \\
\hline $30-1-4-1-1$ & 47.0 & 41.5 & 36.0 & 34.0 & 31.0 & 25.6 & 5.1 & 4.1 & 4.2 & 61.5 & 56.1 & 39.5 & 4.4 & 4.0 & 3.5 & 1.0 & 1.2 & 0.9 \\
\hline $25-1-1-1-4$ & 48.9 & 41.1 & 35.1 & 33.3 & 31.2 & 26.0 & 4.9 & 4.2 & 4.2 & 62.8 & 55.6 & 44.0 & 3.8 & 3.9 & 3.3 & 1.2 & 1.3 & 1.1 \\
\hline $24-2-1-2-1-2$ & 46.8 & 41.9 & 37.8 & 33.5 & 31.1 & 28.8 & 4.4 & 4.1 & 4.1 & 57.7 & 54.2 & 37.6 & 4.3 & 3.9 & 3.4 & 1.3 & 1.4 & 0.9 \\
\hline PI603756 & 44.5 & 41.5 & 40.1 & 27.5 & 31.3 & 30.7 & 3.4 & 2.6 & 2.8 & 54.9 & 54.3 & 55.0 & 3.8 & 3.8 & 2.3 & 1.0 & 1.1 & 1.0 \\
\hline PI587982A & 45.8 & 39.8 & 41.5 & 28.9 & 28.7 & 26.8 & 3.7 & 2.7 & 2.8 & 55.7 & 54.9 & 55.6 & 4.7 & 3.8 & 2.4 & 0.9 & 1.3 & 1.0 \\
\hline \multicolumn{19}{|l|}{ CHECKS } \\
\hline LD00-3309 & 41.0 & 39.6 & 36.0 & 26.6 & 26.0 & 23.9 & 3.2 & 3.0 & 3.4 & 55.1 & 56.3 & 56.1 & 4.7 & 4.1 & 2.9 & 0.9 & 1.1 & 0.9 \\
\hline DT98-9102 & 41.9 & 40.6 & 38.9 & 30.2 & 25.2 & 23.8 & 3.4 & 3.1 & 2.5 & 55.1 & 55.7 & 50.5 & 4.8 & 3.7 & 2.6 & 0.9 & 0.9 & 1.0 \\
\hline DT97-4290 & 43.4 & 41.1 & 38.6 & 26.5 & 28.5 & 26.0 & 3.3 & 2.7 & 2.8 & 60.6 & 54.5 & 43.5 & 4.8 & 3.9 & 2.6 & 0.8 & 1.2 & 0.7 \\
\hline AG4903 & 44.0 & 37.8 & 27.0 & 28.0 & 29.0 & 19.2 & 3.2 & 2.6 & 2.7 & 59.9 & 53.4 & 43.3 & 4.8 & 3.5 & 2.4 & 1.0 & 1.1 & 0.7 \\
\hline $94 \mathrm{~B} 73$ & 43.2 & 33.1 & 26.5 & 27.7 & 25.1 & 19.5 & 3.8 & 2.6 & 2.4 & 56.0 & 37.9 & 44.1 & 4.8 & 3.8 & 2.6 & 1.0 & 1.0 & 1.1 \\
\hline $5601 T$ & 42.2 & 33.1 & 26.2 & 26.6 & 24.9 & 19.5 & 3.5 & 2.7 & 2.9 & 60.1 & 54.7 & 43.2 & 4.8 & 3.5 & 3.0 & 1.0 & 1.1 & 1.0 \\
\hline LSD & 0.81 & 0.91 & 0.62 & 0.74 & 0.83 & 0.58 & 0.22 & 0.33 & 0.24 & 2.26 & 4.56 & 1.49 & 0.19 & 0.18 & 0.19 & 0.58 & 0.07 & 0.058 \\
\hline \multicolumn{19}{|c|}{ SEED QUALITY COMPONENT } \\
\hline Genotype & Germ & Germ & Germ & AA & AA & AA & HS & HS & HS & & & & & & & & & \\
\hline HG (Exotic) & H1 & $\mathrm{H} 2$ & Н3 & H1 & $\mathrm{H} 2$ & H3 & H1 & H2 & H3 & & & & & & & & & \\
\hline $34-3-1-2-4-1$ & 92.0 & 78.7 & 68 & 90.3 & 82.3 & 39.7 & 0.0 & 0.0 & 7.0 & & & & & & & & & \\
\hline $30-1-4-1-1$ & 93.0 & 83.7 & 48.3 & 71.7 & 45.0 & 47.3 & 0.0 & 0.0 & 0.0 & & & & & & & & & \\
\hline $25-1-1-1-1-4$ & 91.0 & 76.0 & 47.0 & 61.0 & 57.3 & 23.0 & 1.3 & 0.0 & 0.0 & & & & & & & & & \\
\hline $24-2-1-2-1-2$ & 90.0 & 63.7 & . & 96.7 & 75.0 & 24.0 & 2.3 & 2.1 & . & & & & & & & & & \\
\hline Pl603756 & 95.7 & 85.5 & 73.0 & 84.0 & 72.7 & 69.5 & 0.0 & 0.5 & 0.0 & & & & & & & & & \\
\hline PI587982A & 93.0 & 81.7 & 59.5 & 68.0 & 52.0 & 27.3 & 1.3 & 1.7 & 0.0 & & & & & & & & & \\
\hline \multicolumn{19}{|l|}{ CHECKS } \\
\hline LD00-3309 & 78.0 & 67.5 & 38.3 & 52.0 & 42.0 & 26.7 & 3.3 & 4.3 & 0.0 & & & & & & & & & \\
\hline DT98-9102 & 78.3 & 63.0 & 53.7 & 48.3 & 50.7 & 29.3 & 8.3 & 3.7 & 0.3 & & & & & & & & & \\
\hline DT97-4290 & 87.7 & 62.7 & 38.3 & 52.0 & 38.3 & 41.3 & 0.0 & 0.3 & 7.0 & & & & & & & & & \\
\hline AG4903 & 87.3 & 61.0 & 47.0 & 55.7 & 40.0 & 12.0 & 0.0 & 4.0 & 0.0 & & & & & & & & & \\
\hline $94 \mathrm{~B} 73$ & 76.3 & 55.3 & 45.3 & 57.3 & 36.3 & 25.3 & 5.7 & 6.7 & 0.0 & & & & & & & & & \\
\hline $5601 T$ & 81.3 & 65.3 & . & 68.0 & 41.0 & 32.0 & 19.3 & 8.3 & 6.7 & & & & & & & & & \\
\hline LSD & 3.13 & 10.8 & 5.02 & 4.28 & 9.94 & 4.56 & 0.87 & 2.13 & 1.46 & & & & & & & & & \\
\hline
\end{tabular}

Plants were grown in a Sharkey clay soil in 2011 at Stoneville, MS, USA.

- Data not available due to the delayed harvest effect. 
was observed when the data were expressed on a genotypic background (exotic line vs. non-exotic check) basis, except that glucose levels did not differ between groups across years (Table 3).

\section{Effect of Delayed Harvest on Germination, Accelerating Aging, and Hard-Seed Traits}

Germination (Germ) rates, evaluated at initial harvest, were higher in three (34-3-1-2-4-1, 30-1-4-1-1, and 25-1-1-1-1-4) of the HG breeding lines, all derived from PI587982A, compared to the non-exotic checks in both 2010 and 2011 (Tables 4, 5). For AA rate, all exotic lines were higher than all non-exotic lines in 2010 at H1 (Table 4). In 2011, all exotic lines, except 30-1-4-11, were higher than all non-exotic lines for AA at H1 (Table 5). Delayed harvesting significantly reduced Germ and AA rates, especially the 28-day delay after initial harvest. Yet in 2010, after a harvest delay of 28 days, 34-3-1-2-4-1 had a higher germination rate $(74 \%)$ and AA rate (52.7\% than any parent or non-exotic line (Table 4). In 2011, after a delayed harvest of 28 days, exotic plant introduction PI603756 had the highest germination rate (73\%) and AA rate (69.5\%) than any other line (Table 5). No consistent trend was found for the effect of delayed harvest on HS. However, all four HG breeding lines had significantly less HS than cultivars LD00-3309, AG4903, and 94B73 at H1 and H2 in 2010 (Table 4).

\section{Responses of Genotypic Backgrounds to Delayed Harvest for Seed Quality}

When the data were expressed based on the two genotypic backgrounds (exotic genotypic background vs. and non-exotic genotypic background), the results showed that seed protein, oleic acid, TB, SCB, Lig, fructose, germination, and accelerated aging were higher in the exotic lines compared to the non-exotic lines (Figure 1). Oil was higher in the non-exotic lines than in the exotic lines. Differences in sucrose were not significant at the $\mathrm{H} 1$ or $\mathrm{H} 2$ harvests; however, it was higher in exotic breeding lines compared to check varieties at 28 after harvest maturity (Figures 1, 2).

\section{Correlations between Germination and Seed Composition and Seed Quality Component Traits}

There were significant positive correlations between germination and protein, stearic, oleic, TB, SCB, and sugars (sucrose, glucose, and fructose) (Table 6). However, negative correlations were observed between germination and yield, oil, linoleic fatty acid, hard-seed, and seed wrinkling. These correlation were obtained when all variables of seed composition components and seed quality components were correlated across all genotypes, harvesting times ( $\mathrm{H} 1, \mathrm{H} 2$, and $\mathrm{H} 3)$, and years. This approach will provide us with a more accurate pattern of changes across the entire genotypic spectrum. These correlations include 216 combined variables. Therefore, a low value of $\mathrm{R}$, such as \pm 0.2 can represent a highly significant correlation $\left(^{* * *}\right)$ between two variables.

\section{DISCUSSION}

\section{Effects of Year, Genotype, Harvesting Time, and Their Interactions}

The significant effects of genotype $(\mathrm{G})$, time of harvest $(\mathrm{H})$, and interactions of $\mathrm{G} \times \mathrm{Y} \times \mathrm{H}$ for protein and fatty acids indicated that these factors are the main driving force behind the differences in these seed nutritional components. The significant interactions of these factors for these constituents indicated that the environmental differences in each year affected these constituents differently, and the response of these constituents at each harvest time was also different. Since the experiment was irrigated, the differences in each year could be due to temperature (Figure 3). For example, weather data (MSUCares, 2017) indicate that the maximum temperature in April was $30^{\circ} \mathrm{C}$ in 2010 and $28^{\circ} \mathrm{C}$ in 2011. In August, the maximum temperature was $37^{\circ} \mathrm{C}$ in 2010 and $35.4^{\circ} \mathrm{C}$ in 2011 . These differences in temperature, especially in August when seed-fill occurs, could be a source of seed quality component differences in each year. Previous research showed that different patterns of temperature in each year affected seed composition differently (Bellaloui et al., 2009, 2017). The non-significant effect of year (Y) for most of the seed quality components indicates that the pattern of these components had a similar effect in each year. The significant effect of genotypic background (GB), $\mathrm{Y}, \mathrm{H}$, and their interactions, when the data were analyzed based on exotic line vs. non-exotic check backgrounds, indicated that the differences in seed quality components of the seed were due to these factors. The interaction effects of these factors for seed quality components indicated that the differences in these components changed each year and that they were probably due to the temperature differences in each year. The response of these components to harvest time depended on each genotypic background, whether exotic or non-exotic.

\section{Effects of Delayed Harvest on Seed Protein, Fatty Acids, Seed Coat B and Lignin, and Sugars}

A recent study evaluated 16 soybean genotypes, among them four breeding lines expressing the HG trait under high heat environments, and showed that these HG lines had high seed protein and oleic acid (Bellaloui et al., 2017). The association between seed protein or oleic acid with germination is still not clear. LeVan et al. (2008) investigated the influence of seed composition and germination under a controlled environment and under field conditions. They concluded that seed composition components, such as protein, oil, and fatty acids, may play a major role in imbibitional injury at low seed moisture content. They also observed a quadratic relationship between seed protein content and seed germination, but suggested further research was needed on the relationship between seed germination and protein, and fatty acids. Recently, Chebrolu et al. (2016) observed low protein synthesis or protein degradation in the heat sensitive genotype DT97-4290 (a non-exotic check in the current study) under high heat compared to the heat tolerant genotype 25-1-1-4-1-1 (a sister 


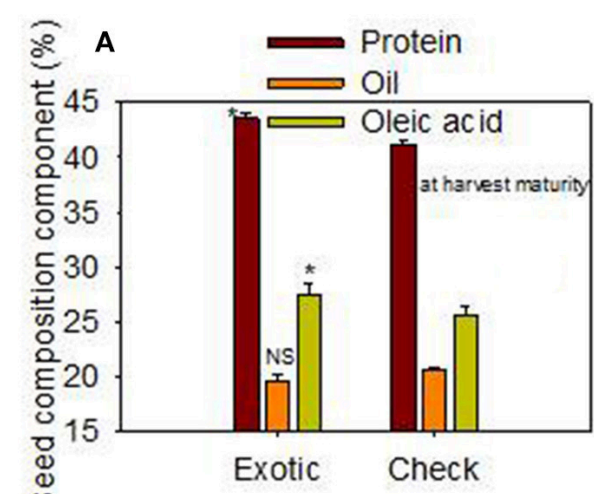

Genotypic background
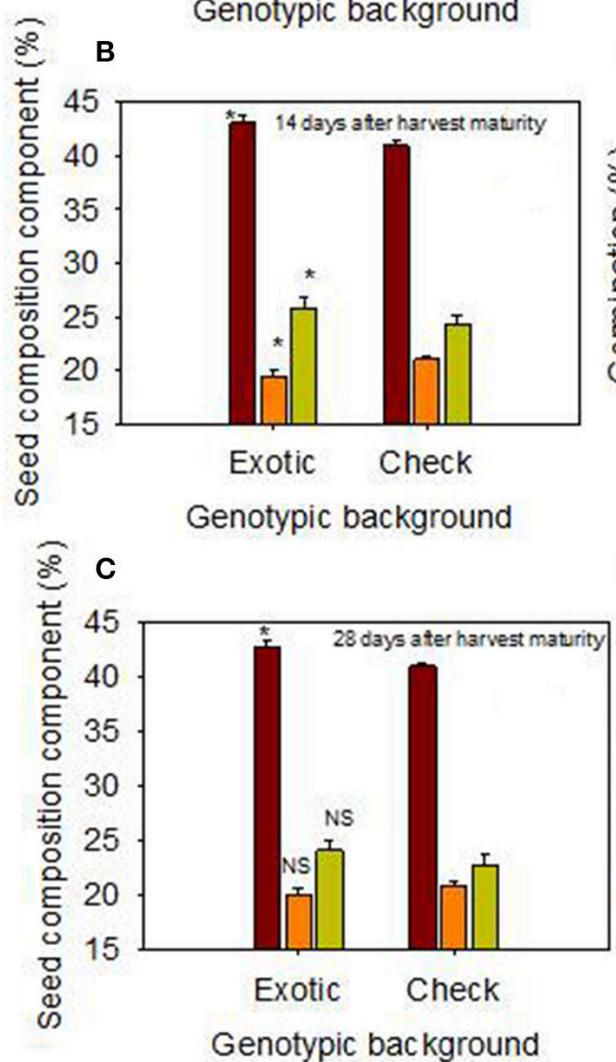

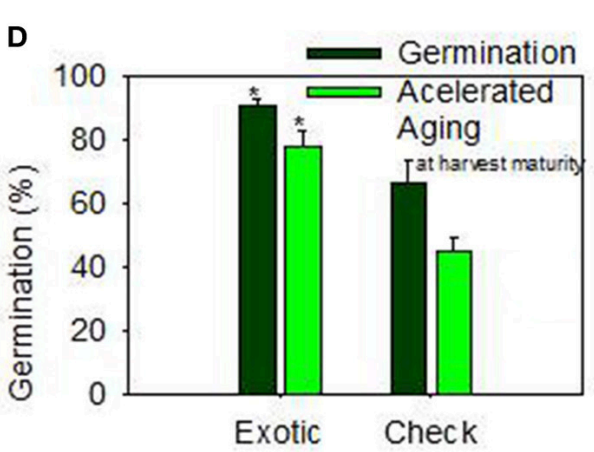

Genotypic background

E

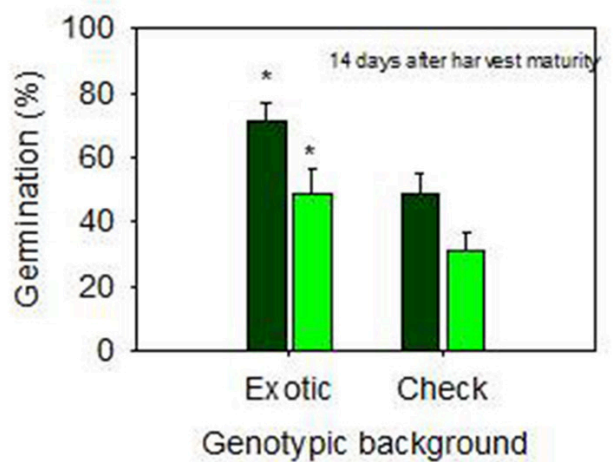

F

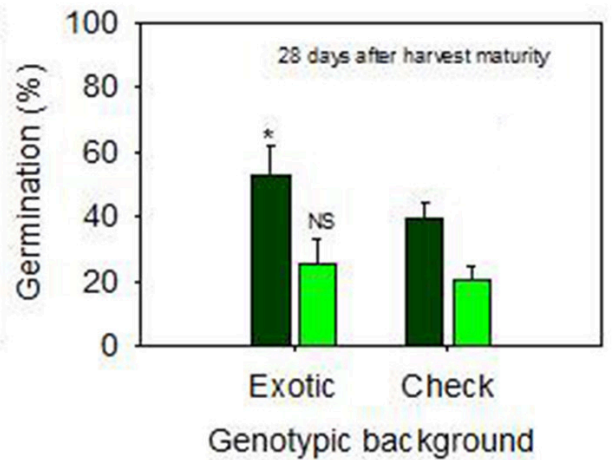

FIGURE 1 | Effect of delayed harvest on seed protein, oil, and oleic acid (\%) at harvest maturity (A), 14 days after harvest maturity (B), and 28 days after harvest maturity (C); and effect of delayed harvest on germination and accelerated aging (\%) at harvest maturity (D), 14 days after harvest maturity (E), and 28 days after harvest maturity (F). Comparison was made between exotic breeding lines and exotic accessions vs. non-exotic checks (Control): genotypic backgrounds comparison. * Means there is a significant difference at $P \leq 0.05$ between similar color bars in each graph. Bars are mean values \pm SE. We used the term "harvest maturity" to refer to the time of harvest shortly after full maturity (R8). The experiment was conducted in 2010 and 2011 in Stoneville, MS, USA.

line of 25-1-1-1-1-4 in the current study), which maintained similar protein levels across multiple temperature environments $\left(28 / 22^{\circ} \mathrm{C}, 36 / 24^{\circ} \mathrm{C}\right.$, and $42 / 26^{\circ} \mathrm{C}$ day/night). A previous study (Bellaloui et al., 2017) involving high field temperatures showed that breeding line 25-1-1-4-1-1 showed higher protein content (43.3 and $40.1 \%$ for 2012 and 2013, respectively) than cultivar AG4903 (38.9 and 37.2\%, respectively. AG4903 is also included in the current study. These observations support the current study, where protein levels of AG4903 were less than those of
HG line 25-1-1-1-1-4 in both 2010 and 2011 and strengthen the notion that HG lines have higher protein levels than lines from the traditional germplasm pool under high heat conditions. The high levels of total $B$ and seed coat $B$ and lignin in the seed coats of HG lines could be due to the role of B and lignin in germination and seed coat protection. It is well established that $\mathrm{B}$ has structural and metabolic roles, being involved in cell wall structure and cell wall and cell membrane integrity. Boron has a role in protecting the seed coat from deterioration by preventing 

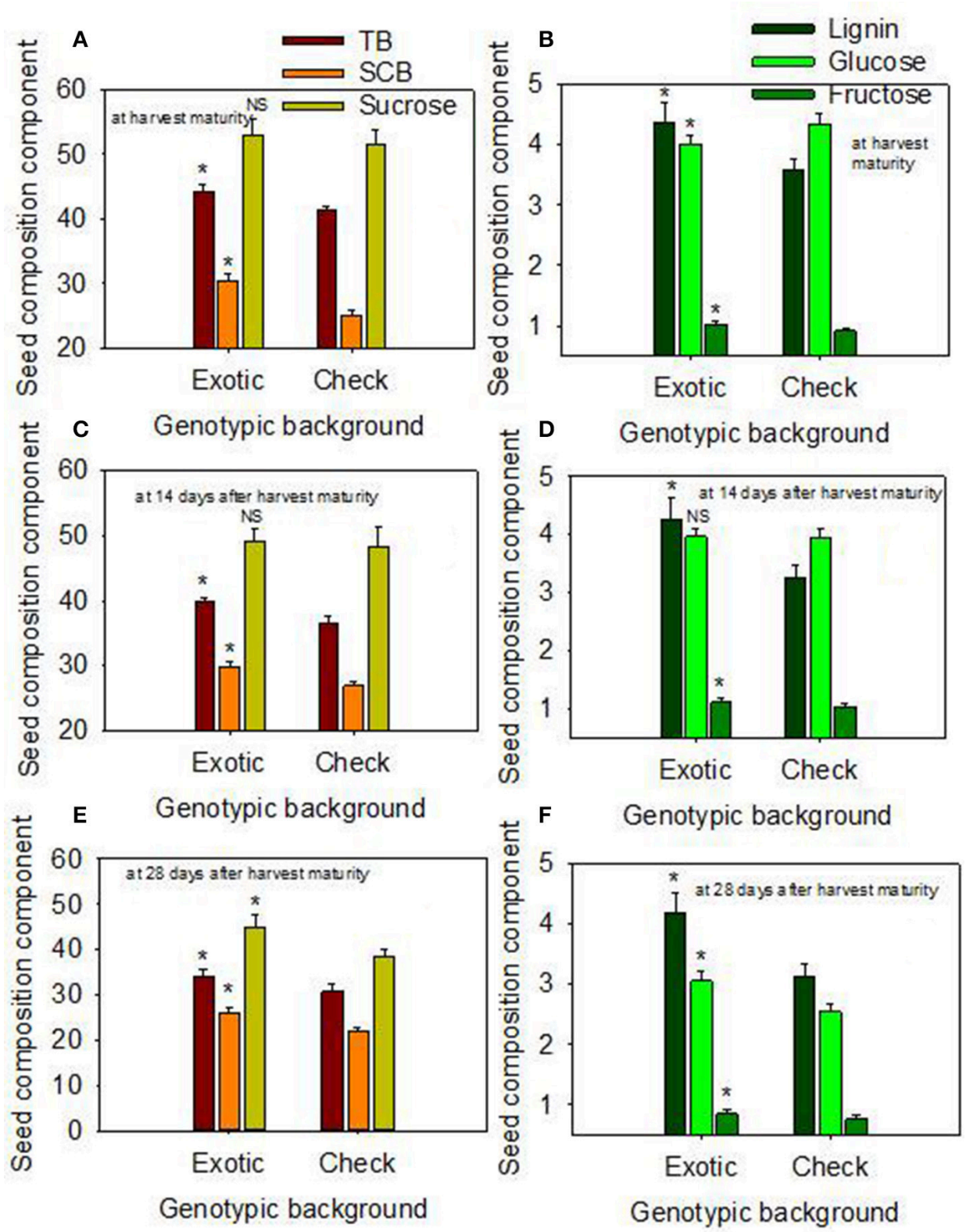

FIGURE 2 | Effect of delayed harvest on total seed boron (TB, $\mathrm{mg} \mathrm{kg}^{-1}$ ), seed coat boron (SCB, $\mathrm{mg} \mathrm{kg}^{-1}$ ), and seed sucrose (mg g ${ }^{-1}$ ) at harvest maturity (A), 14 days after harvest maturity (C), and 28 days after harvest maturity (E); and effect of delayed harvest on seed coat lignin [(mg thioglycolic acid (LTGA) $\mathrm{g}$ dwt ${ }^{-1}$ ), seed glucose $\left(\mathrm{mg} \mathrm{g}^{-1}\right)$, and seed fructose $\left(\mathrm{mg} \mathrm{g}^{-1}\right)$ at harvest maturity (B), 14 days after harvest maturity (D), and 28 days after harvest maturity (F). Comparison was made between exotic breeding lines and exotic accessions vs. non-exotic checks (Control): genotypic backgrounds comparison. * Means there is a significant difference at $P \leq 0.05$ between similar color bars in each graph. Bars are mean values $\pm \mathrm{SE}$. We used the term "harvest maturity" to refer to the time of harvest shortly after full maturity (R8). The experiment was conducted in 2010 and 2011 in Stoneville, MS, USA.

cell wall, cell membrane, and seed coat leaking. A recent study showed significantly higher accumulation of $\mathrm{K}, \mathrm{P}, \mathrm{N}, \mathrm{B}, \mathrm{Cu}$, and Mo in seeds of the two HG/heat tolerant breeding lines 25-1-1-41-1 and 34-3-1-2-4-1, when compared to the other 14 genotypes grown under the high heat of the Mississippi Delta (Bellaloui et al., 2017). HG breeding line 34-3-1-2-4-1 was also included in the current study. This suggests that these nutrients, including $\mathrm{B}$, may play important roles in seed germination. Boron has been considered one of the specific nutrients, in addition to $\mathrm{Ca}$, $\mathrm{K}, \mathrm{Cu}, \mathrm{Fe}, \mathrm{Co}, \mathrm{Zn}$, and $\mathrm{Mn}$, involved in seed coat resistance, phenol metabolism, and lignin biosynthesis, which are involved in membrane permeability and integrity (Marschner, 1995). To our knowledge this is the first report of relating B and lignin levels in the seed coat to germination rates under high heat conditions. Additional research is needed to further clarify these relationships. 

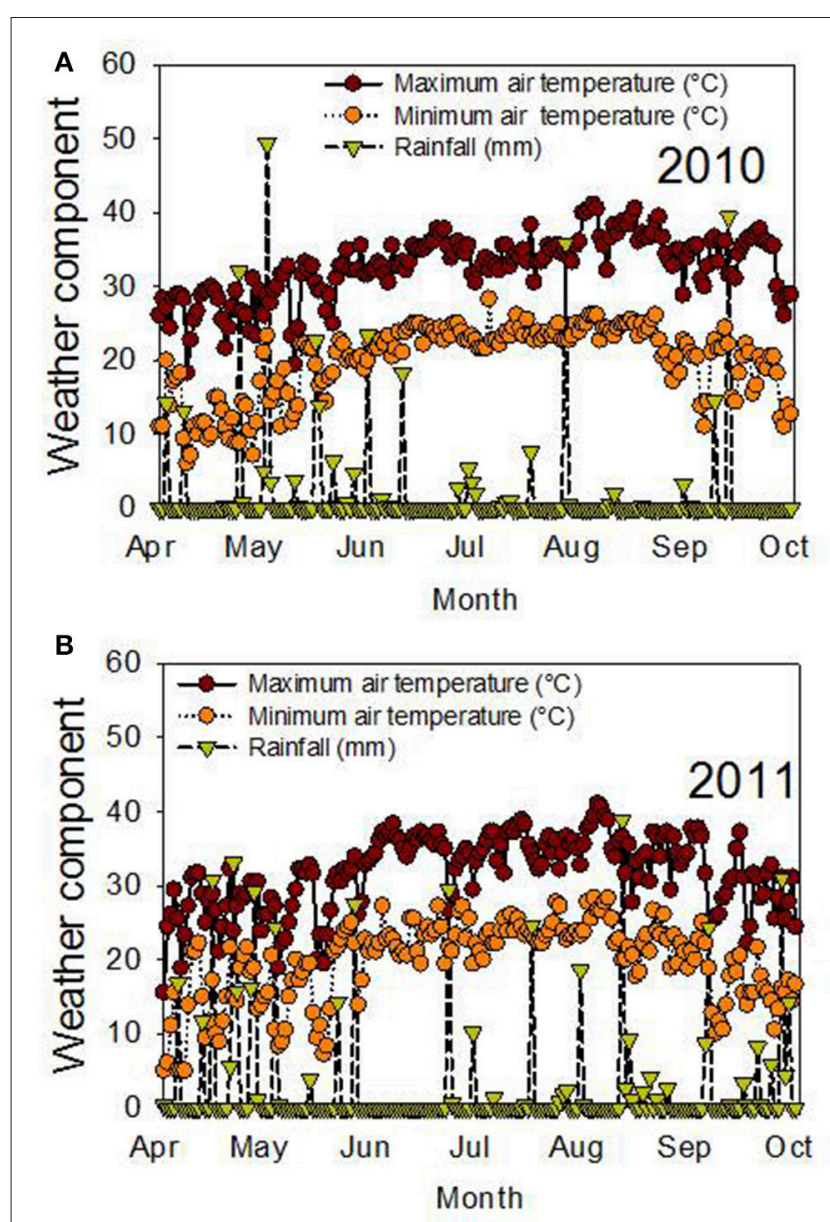

FIGURE 3 | Air temperature $\left({ }^{\circ} \mathrm{C}\right)$ and rainfall $(\mathrm{mm})$ during the growing season in 2010 (A) and 2011 (B). The experiment was conducted in 2010 and 2011 in Stoneville, MS, USA.

The role of seed coat lignin (lig) for seed composition, germination, vigor, and seed and seed coat physical and chemical properties was previously reported by Pereira et al. (1985), Alvarez et al. (1997), Romkaew et al. (2008), Bellaloui et al. (2012b), Baldoni et al. (2013), and de Oliveira et al. (2014). However, in these studies, there were no lines with HG. Therefore, the objective of this research was to advance our knowledge of boron nutrition and further understand the relationships between seed coat B and Lig to seed nutrition and seed germinability under high heat conditions using HG lines. Our results show that all HG lines had high Lig at full maturity, but the content of Lig declined with delays in harvest even in the HG lines, being the lowest at 28 days after initial harvest. However, the content of Lig in the HG lines was significantly higher than that in the non-exotic checks, even after 28 days from initial harvest, indicating that Lig could be associated with seed germination and health of the seed. Seed coat lignin in HG lines was accompanied by significantly less seed coat wrinkling at the $\mathrm{H} 1$ harvest compared with the nonexotic checks, even at 28 days after initial harvest. Seed coat lignin content was different between lines due to genotypic differences.
Although seed sugars did not show clear differences between $\mathrm{HG}$ lines and non-exotic checks at $\mathrm{H} 1$, higher levels of sucrose, glucose, and fructose were shown in HG lines than in the non-exotic checks at delayed harvest as well. This indicates that HG lines maintained higher levels of sugars under stress environments (weathering effects), which may be due to less leakage of these sugars through the seed coat and seed membrane as a result of the higher content of SCB and Lig. Both Lig and SCB were reported to be involved in carbohydrate and phenolic metabolism (Marschner, 1995) to protect cell walls and cell membranes from the physical damage of seed coat deterioration (Pereira et al., 1985; Alvarez et al., 1997; Romkaew et al., 2008; Bellaloui et al., 2012b; Baldoni et al., 2013; de Oliveira et al., 2014), especially under stress environments. The biological functions of the oligosaccharides are not clear (Ren et al., 2009), but other researchers, for example, Obendorf (1997) reported that oligosaccharides and sucrose are important for desiccation tolerance during soybean seed development and maturation, and protection of seeds against damage during seed dehydration and aging. It should be noted that published research has shown that the activity of sucrose synthase in nodules, the main enzyme involved in sucrose hydrolysis, decreased under conditions of drought (González et al., 1995; Streeter, 2003), suggesting higher sensitivity of sucrose to abiotic stress.

\section{Effect of Delayed Harvests on Germination, Accelerating Aging, and Hard-Seed Traits}

The higher Germ and AA in HG lines is expected as these lines were bred for HG. The lower HS rates are also expected as germination is inversely correlated with HS. Previous research showed that high germinating genotypes had the lowest hard seed and seed wrinkling percentages (Smith et al., 2008; Mengistu et al., 2009, 2010; Bellaloui et al., 2012c, 2017). Smith et al. (2008) evaluated 486 accessions, 24 ancestors, and cultivars Stalwart, Croton 3.9, and Stressland with MG ranging from 000 to MG V under field conditions under the ESPS of the midsouthern USA in 2002 and 2003, and a significant negative correlations was observed between standard germination and hard seed, and wrinkled seed, phomopsis, and seed weight. Mengistu et al. (2009) evaluated genotypes under irrigated and non-irrigated conditions and reported that genotypes with higher germination showed lower hard-seed rate and lower phomopsis seed infection. It was earlier reported that high temperatures with alternating periods of wet and dry conditions, such as in the ESPS of the Midsouth, lead to lower germination and increased seed coat wrinkling (Franca-Neto et al., 1993). Similar results were found for seed germination and hard seed by Bellaloui et al. (2012c). Although the cause of poor seed quality in the ESPS is still not fully understood, high temperature, soil moisture, and disease infection, especially by Phomopsis, during seed-fill and pre-harvest lead to hard-seed and low seed viability and vigor (TeKrony et al., 1980; Roy et al., 1994). The differences in Germ, AA, and HS among the genotypes are due to genotypic differences and environmental conditions that vary each year (Smith et al., 2008; Mengistu et al., 2009; Bellaloui et al., 2017). 
TABLE 6 | Correlation (coefficient of correlation, $R$; and significant level, $P$ ) between germination (Germ) and accelerating aging (AA), and seed composition components (protein, P; oil, O; palmitic, Pal; stearic, St; oleic, Ol; linoleic, Lin; linolenic, Lino; hard-seed, HS; shattering, Shat; yield; total boron, TB; cell wall boron, CWB; lignin, Lig; sucrose, Suc; glucose, Glu; and fructose, Fru across years and across genotypes and across three harvesting times (harvest at harvest maturity, shortly after R8 (H1); 14 days after harvest maturity (H2); and 28 days after harvest maturity $(\mathrm{H} 3)$ in soybean breeding lines differing in seed germinability under high heat conditions. Six exotic breeding lines and accessions and six non-exotic checks were used.

\begin{tabular}{|c|c|c|c|c|c|c|c|c|c|c|c|c|c|c|c|c|c|c|}
\hline & $\mathbf{P}$ & 0 & Pal & St & OI & Lin & Lino & Germ & AA & HS & Shat & Yield & TB & CWB & Lig & Suc & Glu & Fru \\
\hline \multirow[t]{2}{*}{ Germ } & $R=0.5$ & -0.5 & -0.0 & 0.3 & 0.5 & -0.5 & -0.0 & 1.0 & 0.7 & -0.5 & -0.1 & -0.4 & 0.6 & 0.5 & 0.3 & 0.5 & 0.4 & 0.3 \\
\hline & $P={ }^{* \star \star}$ & $* * *$ & NS & $* \star \star$ & $* * *$ & $\star * *$ & NS & NA & $* * *$ & $* \star *$ & NS & $* * *$ & $\star * *$ & $* * *$ & 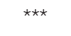 & $* * *$ & $\star * \star$ & $* \star \star$ \\
\hline \multirow[t]{2}{*}{ AA } & $R=0.3$ & -0.3 & -0.2 & 0.3 & 0.6 & -0.6 & 0.03 & 0.7 & 1.0 & -0.2 & -0.2 & -0.4 & 0.6 & 0.6 & 0.19 & 0.5 & 0.4 & 0.4 \\
\hline & $P={ }^{\star \star \star}$ & $\star \star \star \star ~$ & * & $\star \star \star$ & $\star \star \star$ & $\star \star \star \star ~$ & NS & $\star \star \star \star ~$ & $\mathrm{~N}$ & $\star \star \star \star ~$ & * & $\star \star \star$ & $\star \star \star$ & $\star \star \star$ & NS & $\star \star \star \star$ & $\star \star \star \star ~$ & $\star \star \star$ \\
\hline \multirow[t]{2}{*}{ HS } & $R=-0.2$ & 0.2 & 0.0 & -0.2 & -0.3 & 0.3 & 0.0 & -0.5 & -0.2 & 1.0 & -0.1 & 0.2 & -0.1 & -0.3 & -0.1 & -0.2 & 0.01 & -0.2 \\
\hline & $P={ }^{*}$ & * & NS & ** & $* * *$ & $* * *$ & NS & $* \star *$ & $* \star \star$ & NA & * & NS & NS & $* * *$ & NS & * & NS & * \\
\hline \multirow[t]{2}{*}{ Shat } & $R=0.3$ & -0.2 & 0.2 & -0.1 & -0.0 & 0.0 & -0.1 & -0.10 & -0.2 & -0.1 & 1.00 & -0.3 & -0.2 & -0.03 & 0.1 & -0.2 & -0.3 & -0.1 \\
\hline & $P={ }^{* \star \star}$ & $* * *$ & * & NS & NS & NS & NS & NS & * & * & NA & * & $\star * \star$ & NS & NS & * & $* * *$ & NS \\
\hline \multirow[t]{2}{*}{ Wr } & $R=-0.5$ & 0.21 & -0.2 & -0.1 & -0.4 & 0.4 & 0.2 & -0.74 & -0.7 & 0.6 & -0.5 & 0.3 & -0.4 & -0.7 & -0.3 & -0.2 & 0.2 & -0.1 \\
\hline & $P={ }^{* \star \star}$ & NS & NS & NS & $* * *$ & $* * *$ & NS & $* \star \star$ & 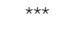 & $* \star \star$ & $* * \star$ & * & $\star \star \star$ & $\star * *$ & $* \star$ & NS & NS & NS \\
\hline \multirow[t]{2}{*}{ Yield } & $R=-0.1$ & 0.2 & 0.7 & -0.6 & -0.0 & 0.1 & -0.4 & -0.4 & -0.4 & 0.2 & -0.3 & 1.00 & -0.6 & -0.3 & 0.4 & -0.8 & -0.5 & -0.2 \\
\hline & $P=\mathrm{NS}$ & NS & 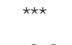 & $\star \star \star *$ & NS & NS & $* \star \star$ & $\star \star \star *$ & $* * *$ & NS & * & NA & $* \star *$ & $\star *$ & $* \star \star$ & $* * \star$ & $* \star \star$ & NS \\
\hline \multirow[t]{2}{*}{ TB } & $R=0.3$ & -0.3 & -0.2 & 0.4 & 0.5 & -0.5 & 0.1 & 0.6 & 0.7 & -0.1 & -0.2 & -0.6 & 1.0 & 0.8 & 0.1 & 0.7 & 0.6 & 0.5 \\
\hline & $P={ }^{\star \star \star}$ & $* * *$ & $* * *$ & $\star * \star$ & $* * *$ & $* * *$ & NS & $* \star *$ & $* * \star$ & NS & $* \star \star$ & $* \star *$ & NA & $\star * \star$ & NS & $* \star \star$ & $* \star \star$ & $* * *$ \\
\hline \multirow[t]{2}{*}{ CWB } & $R=0.5$ & -0.4 & -0.1 & 0.2 & 0.5 & -0.5 & 0.0 & 0.5 & 0.6 & -0.3 & -0.0 & -0.3 & 0.8 & 1.00 & 0.3 & 0.6 & 0.4 & 0.5 \\
\hline & $P={ }^{* \star \star}$ & $* \star *$ & NS & $* \star \star$ & $* * *$ & $* * *$ & NS & $* \star *$ & $* * *$ & $* * *$ & NS & * & $* * *$ & NA & $* \star \star$ & $* \star \star$ & $* \star \star$ & $* * *$ \\
\hline \multirow[t]{2}{*}{ Lig } & $R=0.4$ & -0.3 & 0.6 & -0.3 & 0.2 & -0.2 & -0.3 & 0.3 & 0.1 & -0.1 & 0.1 & 0.4 & 0.1 & 0.3 & 1.0 & 0.0 & 0.2 & 0.0 \\
\hline & $P={ }^{\star \star \star}$ & 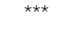 & $\star \star \star \star ~$ & $\star \star \star$ & $\star \star$ & $\star \star *$ & $\star \star \star \star ~$ & $\star \star \star \star ~$ & NS & NS & NS & $\star \star \star$ & NS & $\star \star \star$ & NA & NS & $\star \star$ & NS \\
\hline \multirow[t]{2}{*}{ Suc } & $R=0.3$ & -0.3 & -0.5 & 0.5 & 0.5 & -0.5 & 0.2 & 0.5 & 0.5 & -0.2 & -0.2 & -0.8 & 0.7 & 0.6 & 0.0 & 1.0 & 0.6 & 0.5 \\
\hline & $P={ }^{\star \star \star}$ & 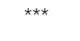 & $\star \star \star ~$ & $\star \star \star$ & $\star \star \star *$ & $\star \star \star *$ & 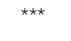 &  & $* \star \star$ & * & * & 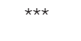 & 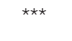 & $\star \star \star$ & NS & & 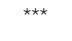 & 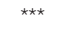 \\
\hline \multirow[t]{2}{*}{ Glu } & $R=0.1$ & -0.1 & -0.1 & 0.2 & 0.4 & -0.3 & -0.1 & 0.4 & 0.4 & 0.0 & -0.3 & -0.5 & 0.6 & 0.4 & 0.2 & 0.6 & 1.00 & 0.4 \\
\hline & $P=\mathrm{NS}$ & * & NS & $\star \star \star$ & $\star * \star$ & $* * \star$ & NS &  & $* \star *$ & NS & $\star \star \star ~$ & $\star \star \star$ & $* \star *$ & $\star \star \star$ & $\star \star \star$ & $\star \star \star *$ & N/A & $\star * \star$ \\
\hline \multirow[t]{2}{*}{ Fru } & $R=0.3$ & -0.3 & -0.3 & 0.3 & 0.4 & -0.4 & 0.2 & 0.3 & 0.4 & -0.2 & -0.1 & -0.2 & 0.5 & 0.5 & 0.00 & 0.5 & 0.4 & 1.00 \\
\hline & $P={ }^{* \star *}$ & $* \star *$ & $* * *$ & $\star * \star$ & $\star * *$ & $\star \star *$ & * & $* \star *$ & $* \star \star$ & $\star \star$ & NS & NS & $\star \star \star$ & $\star * *$ & $\star \star \star *$ & $* \star \star$ & $* \star *$ & NA \\
\hline
\end{tabular}

Plants were grown in a Sharkey clay soil in 2010 and 2011 at Stoneville, MS, USA.

${ }^{\star} P \leq 0.05 ;{ }^{\star \star} P \leq 0.01 ;{ }^{\star \star \star} P \leq 0.001 ;$ NS, not significant; NA, not applicable.

\section{Responses of Genotypic Backgrounds to Delayed Harvest for Seed Quality}

The higher contents of protein, oleic acid, TB, SCB, Lig, fructose, and the higher rates Germ, and AA in HG lines with exotic genotypic backgrounds confirm our hypothesis that these seed nutritional qualities and seed physical and chemical properties of the seed coat could be associated with high Germ and AA in these lines as shown in Figures 1, 2. The higher oil in the non-exotic check background than in the exotic background is due to the inverse relationship between protein and oil (Burton, 1985; Wilcox and Shibles, 2001; Bellaloui et al., 2009). In our analysis, we used two models of statistical analyses. One model was based on the contribution of each genotypes to the variability of the variable studied (seed quality components); the other model was based on the contribution of two categories (exotic vs non-exotic) (genotypic background, GB) to the variability of the seed quality components. Consequently, there were no significant effects of delayed harvest $(\mathrm{H})$ on some seed composition, such as protein, oil, and palmitic and stearic acids when exotic lines vs non-exotic checks were compared, resulting in inconsistent effects of delayed harvest on some seed composition constituents. This may be due to the fact that there must be enough significant variance among individual genotypes to result for an overall significant effect of delayed harvest for some seed constituents. However, when the lines are grouped into only 2 categories (exotic vs non-exotic), the diversity is minimized by comparing only the 2 means.

\section{Correlations between Germination and Seed Composition and Seed Quality Components}

The significant positive correlations between Germ and seed quality components [(protein, stearic, oleic, TB, SCB, and sugars (sucrose, glucose, and fructose)] support our previous observation that these components are involved in germination. Previous research reported that a quadratic relationship between seed protein content and seed germination existed, but the relationship between germination and oil was not conclusive (LeVan et al., 2008). They concluded that further research was needed to better understand the relationship between germination and fatty acids. Also, previous research showed that HG was significantly correlated with seed soluble and structural B (Bellaloui et al., 2009, 2012c, 2017). The negative correlation between germination and HS (Table 6) was also expected as germination and HS are generally inversely correlated because 
seed that cannot imbibe water cannot germinate (Smith et al., 2008; Mengistu et al., 2009; Bellaloui et al., 2017).

\section{CONCLUSION}

The current research confirmed that selecting soybean lines with $\mathrm{HG}$ and heat tolerance resulted in lines with higher protein, oleic acid, sugars, and seed coat B and seed coat lignin, confirming our hypothesis. Higher protein and oleic acid are desirable traits for high soymeal quality and oil stability for shelflife and biodiesel production. High levels of sucrose, glucose, and fructose are desirable for taste and add extra calories to soymeal, such that fewer caloric additives are necessary. Since HG lines had higher seed coat boron and seed coat lignin even at delayed harvest, involvement of seed coat B and seed coat lignin with the HG trait could be a possibility and needs further research. Time of harvest is a major management factor affecting, not only seed production, but also seed nutrition and quality. Both breeding lines 25-1-1-1-4 and 24-2-1-2-1-2 showed competitive high yield in addition to their high seed quality traits.

\section{REFERENCES}

Alvarez, P. J. C., Krzyzanowski, F. C., Mandarino, J. M. G., and Franca-Neto, J. B. (1997). Relationship between soybean seed coat lignin content and resistance to mechanical damage. Seed Sci. Technol. Zurich. 25, 209-214.

AOAC (1990a). "Method 988.05," in Official Methods of Analysis, 15th Edn., ed K. Helrich (Arlington, VA: Elsevier Science Publishing), 70-77.

AOAC (1990b). "Method 920.39," in Official Methods of Analysis, 15th Edn., ed K. Helrich (Arlington, VA: Elsevier Science Publishing), 951.

AOAC (2000). "Method 996.06," in Official Methods of Analysis of AOAC International, 17th Edn., (Rockville, MD: AOAC International), 20-24.

Atabania, A. E., Silitonga, A. S., Badruddin, I. A., Mahlia, T. M. I., Masjuki, H. H., and Mekhilefd, S. (2012). A comprehensive review on biodiesel as an alternative energy resource and its characteristics. Renewable and Sustainable Energy. Reviews 16, 2070- 2093. doi: 10.1016/j.rser.2012.01.003

Baldoni, A., Von Pincho, E. V. R, Fernandes, J. S., Abreu, V. M., and Carvalho, M. L. M. (2013). Gene expression in the lignin biosynthesis pathway during soybean seed development. Genet. Mol. Res. 12, 2618-2624. doi: 10.4238/2013.February.28.2

Belewu, M. A., and Belewu, K. Y. (2007). Comparative physico-chemical evaluation of tiger-nut, soybean and coconut milk sources. Int. J. Agri. Biol. 9, 785-787. Available online at: http://www.fspublishers.org

Bellaloui, N., Brown, P. H., and Dandekar, A. M. (1999). Manipulation of in vivo sorbitol production alters boron uptake and transport in tobacco. Plant Physiol. 119, 735-742. doi: 10.1104/pp.119.2.735

Bellaloui, N., Mengistu, A., Fisher, D. K., and Abel, C. A. (2012a). Soybean seed composition constituents as affected by drought and Phomopsis in phomopsis susceptible and resistant genotypes. J. Crop Improv. 26, 428-453. doi: 10.1080/15427528.2011.651774

Bellaloui, N., Mengistu, A., Walker, E. R., and Young, L. D. (2014). Soybean seed composition as affected by seeding rates and row spacing. Crop Sci. 54, 1782-1795. doi: 10.2135/cropsci2013.07.0463

Bellaloui, N., Mengistu, A., and Zobiole,. L. H. (2012b). Phomopsis seed infection effects on soybean seed phenol, lignin, and isoflavones in maturity group $\mathrm{V}$ genotypes differing in phomopsis resistance. J. Crop Improv. 26, 693-710. doi: $10.1080 / 15427528.2012 .671236$

Bellaloui, N., and Brown, P. H. (1998). Cultivar differences in boron uptake and distribution in celery (Apium graveolens), tomato (Lycopersicon esculentum) and wheat (Triticum aestivum). Plant Soil 198, 153-158. doi: 10.1023/ A: 1004343031242

\section{AUTHOR CONTRIBUTIONS}

NB contributed to the planning, analysis, interpretation, and writing. JS conducted the field portion of the experiment and contributed to the planning, data interpretation, and writing. AM contributed to the analysis, data interpretation, and writing.

\section{ACKNOWLEDGMENTS}

The authors are thankful to Sandra Mosley for lab analysis, and Philip Handly and Hans Hinrichsen for field management. This work was funded by the U.S. Department of Agriculture, Agricultural Research Service Project 6066-21220-012-00D and by United Soybean Board project 9232: "Identification and utilization of exotic germplasm to improve soybean productivity" (Agreement number 58-3611-8-462). Mention of trade names or commercial products in this publication is solely for the purpose of providing specific information and does not imply recommendation or endorsement by the U.S. Department of Agriculture. USDA is an equal opportunity provider and employer.

Bellaloui, N., Reddy, K. N., Gillen, A. M., and Abel, C. A. (2010a). Nitrogen metabolism and seed composition as influenced by foliar boron application in soybean. Plant Soil 336, 143-155. doi: 10.1007/s11104-0100455-6

Bellaloui, N., Smith, J. R., Gillen, A. M., and Ray, J. D. (2010b). Effect of maturity on seed sugars as measured on near-isogenic soybean (Glycinemax) lines. Crop Sci. 50, 1978-1987. doi: 10.2135/cropsci2009.10.0596

Bellaloui, N., Smith, J. R., Gillen, A. M., Fisher, D. K., and Mengistu, A. (2012c). Effect of shade on seed protein, oil, fatty acids, and minerals in soybean lines varying in seed germinability in the early soybean production system. Am. J. Plant Sci. 3, 84-95. doi: 10.4236/ajps.2012.31008

Bellaloui, N., Smith, J. R., Gillen, A. M., and Ray, J. D. (2011). Effects of maturity, genotypic background, and temperature on seed mineral composition in nearisogenic soybean lines in the early soybean production system. Crop Sci. 51, 1161-1171. doi: 10.2135/cropsci2010.04.0187

Bellaloui, N., Smith, J. R., Mengistu, A., Ray, J. D., and Gillen, A. M. (2017). Evaluation of exotically-derived soybean breeding lines for seed yield, germination, damage, and composition under dryland production in the midsouthern USA. Front. Plant Sci. 8:176. doi: 10.3389/fpls.2017. 00176

Bellaloui, N., Smith, J. R., Ray, J. D., and Gillen, A. M. (2009). Effect of maturity on seed composition in the early soybean production system as measured on near-isogenic soybean lines. Crop Sci. 49, 608-620. doi: 10.2135/cropsci2008. 04.0192

Biermann, C. J., and McGinnis, G. D. (1989). Analysis of Carbohydrates by GLC and MS. Boca Raton, FL: CRC Press.

Boydak, E., Alpaslan, M., Hayta, M., Gerçek, S., and Simsek, M. (2002). Seed composition of soybeans grown in the Harran region of Turkey as affected by row spacing and irrigation. J. Agric. Food Chem. 50, 4718-4720. doi: 10.1021/jf0255331

Brown, P. H., Bellaloui, N., Dandekar, A. M., and Hu, H. (1999), Transgenically enhanced sorbitol synthesis facilitates phloem boron transport and increases tolerance of tobacco to boron deficiency. Plant Physiol. 119, 17-20. doi: 10.1104/pp.119.1.17

Brown, P. H., Bellaloui, N., Wimmer, M. A., Bassil, E. S., Ruiz, J., Hu, H., et al. (2002). Boron in plant biology. Plant Biol. 4, 205-223. doi: 10.1055/s-2002-25740

Burton, J. W. (1985). "Breeding soybean for improved protein quantity and quality," in World Soybean Research Conference III: Proceedings, ed R. Shibles, (Ames, IA), 12-17 Aug. 1984, (Boulder, CO: Westview Press), 361-367. 
Chebrolu, K. K., Fritschi, F. B., Ye, S., Krishnan, H. B., Smith, J. R., and Gillman, J. D. (2016). Impact of heat stress during seed development on soybean seed metabolome. Metabolomics 12, 1-14. doi: 10.1007/s11306-0150941-1

Churms, S. C., Zweig, G., and Sherma, J. (1982). Handbook of Chromatography. Boca Raton, FL: CRC Press.

de Oliveira, C. M. G., Kryzanowski, F. C., de Oliveira, M. C. N., and de Barros França-Neto, J., and Henning, A. A. (2014). Relationship between pod permeability and seed quality in soybean. J. Seed Sci. 36, 273-281. doi: 10.1590/2317-1545v36n3919

Diers, B. W., Cary, T. R., Thomas, D. J., and Nickell, C. D. (2006). Registration of 'LD00-3309'soybean. Crop Sci. 46:1384. doi: 10.2135/cropsci2005.06.0164

Dordas, C. (2006). Foliar boron application improves seed set, seed yield, and seed quality of alfalfa. Agron. J. 98, 907-913. doi: 10.2134/agronj2005.0353

Dordas, C., Apostolides, G., and Goundra, O. (2007). Boron application affects seed yield and seed quality of sugar beets. J. Agric. Sci. 145, 377-384. doi: $10.1017 /$ S0021859607006879

FAOSTAT (2011). Production Crops: Faostat.fao.org (2011). AMIS data. Available online at: http://statistics.amis-outlook.org/data/index.html\#COMPARE (Accessed June 26, 2017).

Fehr, W. R., and Caviness, C. E. (1977). Stages of Soybean Development. Iowa Agriculture Experiment Station Special Report 80. Iowa State University, (Ames, IA).

Ferrarese, M. L. L., Zottis, A., and Ferrarese-Filho, O. (2002). Protein-free lignin quantification in soybean (Glycine max) roots. Biologia 57, 541-543. Available online at: http://agris.fao.org/agris-search/search.do?recordID=SK2002000611

Ferrari, R. A., Oliveira, V. S., and Scabio, A. (2005). Oxidative stability of biodiesel from soybean oil fatty acid ethyl esters. Sci. Agric. 62, 291-295. doi: 10.1590/S0103-90162005000300014

Franca-Neto, J. B., Krzyzanowski, F. C., Henning, A. A., West, S. H., and Miranda, L. C. (1993). Soybean seed quality as affected by shriveling due to heat and drought stress during seed filling. Seed Sci. Technol. 21, 107-116.

Franca-Neto, J. B., Krzyzanowski, F. C., West, S. H., and Vaughan, W. R. (1988). Multiple quality evaluation of soybean seed produced in Florida. Soil Crop Sci. Soc. Fla. Proc. 47, 201-206.

Goldbach, H. (1985). Influence of boron nutrition on net uptake and efflux of $32 \mathrm{P}$ and 14C-glucose in Helianthus annuus roots and cell cultures of Daucus carota. J. Plant Physiol. 118, 431-438. doi: 10.1016/S0176-1617(85) 80203-0

González, E. M., Gordon, A. J., James, C. L., and Arrese-Igor, C. (1995). The role of sucrose synthase in the response of soybean nodules to drought. J. Exp. Bot. 46, 515-1,523. doi: $10.1093 / \mathrm{jxb} / 46.10 .1515$

Hu, H., and Brown, P. H. (1994). Localization of boron in cell walls of squash and tobacco and its association with pectin: Evidence for a structural role of boron in the cell wall. Plant Physiol. 105, 681-689. doi: 10.1104/pp.105. 2.681

Jaureguy, L. M., Rodriguez, F. L., Zhang, L., Chen, P., Brye, K., Oosterhuis, D., et al. (2013). Planting date and delayed harvest effects on soybean seed composition. Crop Sci. 53, 2162-2175. doi: 10.2135/cropsci2012. 12.0683

John, M. K., Chuah, H. H., and Neufeld, J. H. (1975). Application of improved azomethine-Hmethod to the determination of boron in soils and plants. Anal. Lett. 8, 559-568. doi: 10.1080/00032717508058240

Krzyzanowski, F. C., Franca-Neto, J. B., Mandarino, J. M. G., and Kaster, M. (2001). Comparison between two gravimetric methods to determine the lignin content in soybean seed coat. Seed Sci. Technol. 29, 619-624.

Krzyzanowski, F. C., Franca-Neto, J. B., Mandarino, J. M. G., and Kaster, M. (2008). Evaluation of lignin content of soybean seed coat stored in a controlled environment. Rev. Bras. Sementes. 30, 220-223. doi: 10.1590/S0101-31222008000200028

LeVan, N. A., Goggi, A. S., and Mullen, R. (2008). Improving the reproducibility of the soybean standard germination test. Crop Sci. 48, 1933-1940. doi: $10.2135 /$ cropsci2007.06.0372

Lohse, G. (1982). Microanalytical azomethine-H method for boron determination in plant tissue. Commun. Soil Sci. Plant Anal. 13, 127-134. doi: 10.1080/00103628209367251

Marschner, H. (1995). Mineral Nutrition of Higher Plants. San Diego, CA: Academic Press, 379-396.
Mengistu, A., Castlebury, L. A., Smith, J. R., Ray, J. D., and Bellaloui, N. (2009). Seasonal progress of phomopsis longicolla infection on soybean plant parts and its relatioship to seed quality. Plant Dis. 93, 1009-1018. doi: 10.1094/PDIS-93-10-1009

Mengistu, A., and Heatherly, L. G. (2006). Planting date, irrigation, maturity group, year, and environment effects on Phomopsis longicolla, seed germination, and seed health rating of soybean in the early soybean production system of the mid-southern USA. Crop Prot. 25, 310-317. doi: 10.1016/j.cropro.2005.05.011

Mengistu, A., Smith, J. R., Bellaloui, N., Paris, R. L., and Wrather, J. A. (2010). Irrigation and time of harvest effects on evaluation of selected soybean accessions against Phomopsis longicolla. Crop Sci. 50, 2055-2064. doi: $10.2135 /$ cropsci2009.11.0657

MSUCares (2017). Mississippi State University, Extension service. Available online at: http://ext.msstate.edu/anr/drec/weather.cgi

Obendorf, R. L. (1997). Oligosaccharides and galactosyl cyclitols in seed desiccation tolerance. Seed Sci. Res. 7, 63-74. doi: 10.1017/S09602585000 $0341 \mathrm{X}$

Pantalone, V. R., Allen, F. L., and Landau-Ellis, D. (2003). Registration of '5601T' soybean. Crop Sci. 43, 1123-1124. doi: 10.2135/cropsci2003.1123

Paris, R. L., Mengistu, A., Tyler, J. M., and Smith, J. R. (2006). Registration of soybean germplasm line DT97-4290 with moderate resistance to charcoal rot. Crop Sci. 46, 2324-2325. doi: 10.2135/cropsci2005.09.0297

Pereira, L. A. G., Franca-Neto, J. B., Costa, N. P., Henning, A. A., and Magalhaes, C. V. (1985). "Teste de metodologia para identificacao de genotipos de alta qualidade fisiologica de sementes de soja," in EMBRAPA. Centro Nacional de Pesquisa de Soja (Londrina, PR). PR. Resultados de Pesquisa de Soja 1984/1985, eds N. P. Costa, J. B. Franca-Neto, and F. C. Krzyzanowski (Londrina: Embrapa Soja), 407-408

Pilbeam, D. J., and Kirkby, E. A. (1983). The physiological role of boron in plants. J. Plant Nutr. 6, 563-582. doi: 10.1080/01904168309363126

Ren, C., Bilyeu, K. D., and Beuselinck, P. R. (2009). Composition, vigor, and proteome of mature soybean seeds developed under high temperature. Crop Sci. 49, 1010-1022. doi: 10.2135/cropsci2008.05.0247

Romkaew, J., Nagaya, Y., Goto, M., Suzuki, K., and Umezaki, T. (2008). Pod dehiscence in relation to chemical components of pod shell in soybean. Plant Produ. Sci. 11, 278-282. doi: 10.1626/pps.11.278

Roy, K. W., Keith, B. C., and Andrews, C. H. (1994). Resistance of hardseeded soybean lines to seed infection by Phomopsis, other fungi, and soybean mosaic virus. Can. J. Plant Pathol. 16, 122-128. doi: 10.1080/07060669409 500769

Sakthivelu, G., Devi, M. K. A., Giridhar, P., Rajasekaran, T., Ravishankar, G. A., Nikolova, M. T., et al. (2008). Isoflavone composition, phenol content, and antioxidant activity of soybean seeds from India and Bulgaria. J. Agric. Food Chem. 56, 2090-2095. doi: 10.1021/jf07 2939 a

Santos, E. M., Piovesan, N. D., Everaldo Gonçalves de Barros, E., and Moreira, M. A. (2013). Low linolenic soybeans for biodiesel: characteristics, performance and advantages. Fuel 104, 861-864. doi: 10.1016/j.fuel.2012. 06.014

Shelp, B. J. (1993). "Physiology and biochemistry of boron in plants," in Boron and Its Role in Crop Production, ed U. C. Gupta (Boca Raton, FL: CRC Press), 53-85.

Smith, J. R., Mengistu, A., Nelson, R. L., and Paris, R. L. (2008). Identification of soybean accessions with high germinability in high-temperature environments. Crop Sci. 48, 2279-2288. doi: 10.2135/cropsci2008.01.0026

Streeter, J. G. (2003). Effects of drought on nitrogen fixation in soybean root nodules. Plant Cell Environ. 26, 1199-1204. doi: 10.1046/j.1365-3040.2003. 01041.x

TeKrony, D. M., Egli, D. B., Balles, J., Tomes, L., and Stuckey, R. E. (1980) Effect of harvest maturity on soybean seed quality and Phomopsis sp. seed infection. Crop Sci. 24, 189-193. doi: 10.2135/cropsci1984.0011183X0024000 $10045 \mathrm{x}$

TeKrony, D. M., Grabau, L. J., DeLacy, M., and Kane, M. (1996). Early planting of early-maturing soybean: effects on seed germination and Phomopsis infection. Agron. J. 88, 428-433. doi: 10.2134/agronj1996.000219620088000 $30011 x$

Wilcox, J. R., Laviolette, F. A., and Athow, K. L. (1974). Deterioration of soybean seed quality associated with delayed harvest. Plant Dis. Rep. 58, 130-133. 
Wilcox, J. R., and Shibles, R. M. (2001). Interrelationships among seed quality attributes in soybean. Crop Sci. 41, 11-14. doi: 10.2135/cropsci2001.41111x

Wilson, R. F. (2004). "Seed composition," in Soybeans: Improvement, Production, and Uses, eds H. Boerma and J. E. Specht (Madison, WI: American Society of Agronomy, Crop Science Society of America, and Soil Science Society of America), 621-668.

Woodstock, L. W., Furman, K., and Leffler, H. R. (1985). Relationship between weathering deterioration and germination, respiratory metabolism, and mineral leaching from cottonseeds. Crop Sci. 25, 459-466, doi: $10.2135 /$ cropsci1985.0011183X002500030008x
Conflict of Interest Statement: The authors declare that the research was conducted in the absence of any commercial or financial relationships that could be construed as a potential conflict of interest.

Copyright (๑) 2017 Bellaloui, Smith and Mengistu. This is an open-access article distributed under the terms of the Creative Commons Attribution License (CC BY). The use, distribution or reproduction in other forums is permitted, provided the original author(s) or licensor are credited and that the original publication in this journal is cited, in accordance with accepted academic practice. No use, distribution or reproduction is permitted which does not comply with these terms. 